\title{
Chemical tagging with APOGEE: discovery of a large population of N-rich stars in the inner Galaxy
}

Ricardo P. Schiavon, ${ }^{1 \star}$ Olga Zamora, ${ }^{2,3}$ Ricardo Carrera, ${ }^{2,3}$ Sara Lucatello, ${ }^{4 \star}$ A. C. Robin, ${ }^{5}$ Melissa Ness, ${ }^{6}$ Sarah L. Martell, ${ }^{7 \star}$ Verne V. Smith, ${ }^{8,9}$ D. A. García-Hernández, ${ }^{2,3}$ Arturo Manchado, ${ }^{2,3,10}$ Ralph Schönrich, ${ }^{11}$ Nate Bastian, ${ }^{1}$ Cristina Chiappini, ${ }^{12}$ Matthew Shetrone, ${ }^{13}$ J. Ted Mackereth, ${ }^{1}$ Rob A. Williams, ${ }^{1}$ Szabolcs Mészáros, ${ }^{14}$ Carlos Allende Prieto, ${ }^{2,3}$ Friedrich Anders, ${ }^{12}$ Dmitry Bizyaev, ${ }^{15,16}$ Timothy C. Beers,${ }^{17}$ S. Drew Chojnowski, ${ }^{18}$ Katia Cunha,,${ }^{8,19}$ Courtney Epstein, ${ }^{20}$ Peter M. Frinchaboy, ${ }^{21}$ Ana E. García Pérez, ${ }^{2}$ Fred R. Hearty, ${ }^{22}$ Jon A. Holtzman, ${ }^{23}$ Jennifer A. Johnson, ${ }^{20}$ Karen Kinemuchi, ${ }^{15}$ Steven R. Majewski, ${ }^{18}$ Demitri Muna, ${ }^{20}$ David L. Nidever, ${ }^{24,25,26}$ Duy Cuong Nguyen, ${ }^{27}$

Robert W. O'Connell, ${ }^{18}$ Daniel Oravetz, ${ }^{15}$ Kaike Pan, ${ }^{15}$ Marc Pinsonneault, ${ }^{20}$ Donald P. Schneider, ${ }^{22}$ Matthias Schultheis, ${ }^{28}$ Audrey Simmons, ${ }^{15}$ Michael F. Skrutskie, ${ }^{18}$ Jennifer Sobeck, ${ }^{18}$ John C. Wilson ${ }^{18}$ and Gail Zasowski ${ }^{29}$

Affiliations are listed at the end of the paper

Accepted 2016 August 31. Received 2016 August 23; in original form 2015 September 11

\begin{abstract}
Formation of globular clusters (GCs), the Galactic bulge, or galaxy bulges in general is an important unsolved problem in Galactic astronomy. Homogeneous infrared observations of large samples of stars belonging to GCs and the Galactic bulge field are one of the best ways to study these problems. We report the discovery by APOGEE (Apache Point Observatory Galactic Evolution Experiment) of a population of field stars in the inner Galaxy with abundances of $\mathrm{N}$, $\mathrm{C}$, and $\mathrm{Al}$ that are typically found in GC stars. The newly discovered stars have high $[\mathrm{N} / \mathrm{Fe}]$, which is correlated with $[\mathrm{Al} / \mathrm{Fe}]$ and anticorrelated with $[\mathrm{C} / \mathrm{Fe}]$. They are homogeneously distributed across, and kinematically indistinguishable from, other field stars within the same volume. Their metallicity distribution is seemingly unimodal, peaking at $[\mathrm{Fe} / \mathrm{H}] \sim-1$, thus being in disagreement with that of the Galactic GC system. Our results can be understood in terms of different scenarios. N-rich stars could be former members of dissolved GCs, in which case the mass in destroyed GCs exceeds that of the surviving GC system by a factor of $\sim 8$. In that scenario, the total mass contained in so-called 'first-generation' stars cannot be larger than that in 'second-generation' stars by more than a factor of $\sim 9$ and was certainly smaller. Conversely, our results may imply the absence of a mandatory genetic link between 'second-generation' stars and GCs. Last, but not least, N-rich stars could be the oldest stars in the Galaxy, the by-products of chemical enrichment by the first stellar generations formed in the heart of the Galaxy.
\end{abstract}

Key words: stars: abundances - stars: chemically peculiar-Galaxy: abundances-Galaxy: bulge-globular clusters: general-Galaxy: halo. 


\section{INTRODUCTION}

While central to our understanding of the formation of the Galaxy, the birthplaces of the stars that make up its main components are not well known. In the case of the Galactic halo, this classical problem (Eggen, Lynden-Bell \& Sandage 1962; Searle \& Zinn 1978) has been framed in modern times within the context of galaxy formation theory (White \& Rees 1978; Blumenthal et al. 1984) in a $\Lambda$ cold dark matter universe (Spergel et al. 2003). Recent evidence that the Galactic halo is split into an inner and an outer component, with distinct chemical compositions (Carollo et al. 2007, 2010; An et al. 2015; Fernández-Alvar et al. 2015), goes along with theoretical predictions for the origin of those components (e.g. McCarthy et al. 2012; Tissera et al. 2014), at least in a qualitative sense (although see Schönrich, Asplund \& Casagrande 2014, for an alternative view). Most importantly, both data and models have reached a degree of sophistication that allows one to begin addressing detailed questions about the nature of the original star-forming units that gestated the stars seen in the halo today - in particular their characteristic masses (e.g. Deason, Belokurov \& Weisz 2015; Fiorentino et al. 2015).

Regarding the bulge, the situation is considerably less clear, which is due partly to difficult observational access to the inner Galaxy, and partly to the short dynamical time-scales, which caused signatures of the early stellar systems to be erased from phase space long ago. Moreover, the physical overlap of all components of the Galaxy (halo, thin and thick discs, bar, and bulge) within its inner few kpc makes a definition of the pertinence of a given star or stellar group to any of those components quite difficult, making the very definition of the bulge itself somewhat contentious. The literature on the Galactic bulge is sufficiently vast to render any attempt at a summary here quite vain. However, the picture emerging from even a brief examination of the state of the art is that of a current lack of a unique definition of the nature of the bulge, both in terms of the distribution of its components in phase space and, to a lesser extent, in terms of its stellar population content. We therefore use the term 'bulge' somewhat loosely, without necessarily implying the existence of a classical spheroidal structure detached from the inner halo, but simply referring to the aggregate of all stellar mass cohabiting the central few kpc of the Galaxy.

Studies of stellar bulge spatial distribution, kinematics, metallicity distribution function (MDF), metallicity gradients, and abundance patterns paint a complex picture. Several groups have confirmed the presence of a complex metallicity distribution of bulge stellar populations (e.g. Zoccali et al. 2008; Hill et al. 2011; Johnson et al. 2011; Dékány et al. 2013; Ness, Freeman \& Athanassoula 2013a; Rojas-Arriagada et al. 2014), with the presence of multiple components, each with characteristic structure and kinematics. At high metallicity $([\mathrm{Fe} / \mathrm{H}] \gtrsim-0.5)$, the bulge appears to be dominated by a boxy/peanut-shaped structure, associated with a bar (Blitz \& Spergel 1991), which in projection has been found to assume an $\mathrm{X}$ shape in $2 \mathrm{MASS}^{1}$ maps (McWilliam \& Zoccali 2010; Nataf et al. 2010; Ness, Freeman \& Athanassoula 2012). On the other hand, stars in the low-metallicity end take on a more spheroidal distribution and are thought to be associated with either the thick disc or halo (e.g. Babusiaux et al. 2010; Ness, Freeman \& Athanassoula 2013a,b; Rojas-Arriagada et al. 2014) or perhaps even a classical spheroidal bulge (e.g. Babusiaux et al. 2010; Hill et al. 2011), although the existence of the latter has been called into question by model fits to stellar counts in the 2MASS and SDSS

${ }^{1}$ 2MASS is the Two Micron All Sky Survey (Skrutskie et al. 2006). catalogues (Robin et al. 2014). Evidence from kinematics pointing to the existence of an anticorrelation between metallicity and velocity dispersion (e.g. Johnson et al. 2011, 2014) jibes well with the above picture. More detailed studies, based on high-quality radial velocities for samples of many thousand stars, show that, while the metal-rich bulge population rotates cylindrically, the kinematics of metal-poor stars is consistent with a slowly rotating spheroid, possibly due to a combination of thick disc and halo (Ness et al. 2013b, 2016). Finally, detailed chemical abundance studies showed, since the early work by McWilliam \& Rich (1994), that metal-poor stars tend to be $\alpha$-enhanced, as in the thick disc and halo, whereas their metal-rich counterparts have $[\alpha / \mathrm{Fe}]$ close to solar (see also Hill et al. 2011; Ness et al. 2013a; Johnson et al. 2014; Ryde et al. 2016). When studied individually, $\alpha$ elements were found not to follow all exactly the same trend with metallicity (Fulbright, McWilliam \& Rich 2007) and, perhaps most importantly, to be slightly more enhanced in metal-rich bulge stars than in their thin- and thick-disc counterparts (Johnson et al. 2014).

One possible approach to gain insights into the nature of starforming units that gave origin to the stars in the Galaxy is through chemical tagging (Freeman \& Bland-Hawthorn 2002; Ting, Conroy \& Goodman 2015; Ting, Conroy \& Rix 2016). The method consists of using very detailed and accurate chemical compositions to identify stars sharing a common origin, with the hope of tracing them back to their original star-forming units. In principle, chemical tagging can be extremely powerful, provided that each and every star-forming unit was characterized by anique detailed abundance pattern. If this assumption is correct, one could distinguish every single star-forming unit from all others by determining a large enough number of elemental abundances. That, of course, is observationally very costly. A weaker, less expensive, form of the method consists of associating a given abundance pattern not necessarily with a unique star-forming unit, but with an entire class - say, stellar clusters above a given mass. In order to work, this weak chemical tagging requires knowledge of a smaller number of elemental abundances than does standard strong chemical tagging.

An early application of weak chemical tagging was pursued by Martell \& Grebel (2010) and Martell et al. (2011, see also Carretta et al. 2010; Lind et al. 2015). These authors discovered halo field stars with very high nitrogen and relatively low carbon abundances, which is an abundance pattern characteristic of some particular globular cluster (GC) populations. On the theory that these stars were originally formed in GCs, Martell and collaborators concluded that they resulted from the dissolution of GCs - a claim that is in line with detections of tidal tails around GCs such as Palomar 5 (Odenkirchen et al. 2003) and NGC 5466 (Belokurov et al. 2006a). By accounting for the expected fraction of GC stars with normal N abundances, Martell et al. (2011) estimated that at least 17 per cent of the stellar mass in the Galactic halo resulted from the dissolution of GCs and/or their parent systems. Based on the same results, but adopting a different set of assumptions, Gratton, Carretta \& Bragaglia (2012) estimated that most of the halo has in fact originated from those systems. Following yet another approach based on a model of the chemical and dynamical evolution of the Galactic GC system, Schaerer \& Charbonnel (2011) estimate that up to 10-20 per cent of the Galactic halo mass was contributed by tidal evaporation of Galactic GCs.

One major difference in these estimates is that they assign different theoretically motivated ratios between the numbers of 'enriched' and 'normal' stars. Our lack of a firm handle on the origin of the multiple-population phenomenon in Galactic GCs is therefore an important limiting factor. Moreover, uncertainties about the shape 
of the initial mass function (IMF) of the Galactic GC system also play a role. The presence of large spreads of elemental abundances, and anticorrelations thereof, in GC stars has been long known (e.g. Norris \& Zinn 1977; Norris \& Cottrell 1979; Da Costa \& Cottrell 1980) and consistently confirmed by more recent observations (e.g. Carretta et al. 2009; Mészáros et al. 2015). ${ }^{2}$ In the past decade, abundance spreads were ascribed to the clusters' intrinsic chemical evolution, either due to some form of feedback-regulated star formation history (e.g. Decressin et al. 2007; D'Ercole et al. 2008; Renzini 2008; Conroy \& Spergel 2011) or to other processes (e.g. Bastian et al. 2013; Hopkins 2014). However, none of the existing models put forth so far can account for the existing chemical composition data in detail (Bastian, Cabrera-Ziri \& Salaris 2015). Yet, the different models make vastly different predictions. In particular, models that propose chemical evolution through feedback-regulated star formation postulate that GCs were 10-100 times more massive in the past (for discussion and references, see, e.g., Gratton et al. 2012; Bastian \& Lardo 2015; Cabrera-Ziri et al. 2015). Moreover, according to these models, the vast majority of this mass must have been in the form of first-generation ${ }^{3}$ stars (henceforth FG stars) which so far remain chemically indistinguishable from field stars of the same $[\mathrm{Fe} / \mathrm{H}]$. These conditions are required so that, for any reasonable IMF, early stellar generations can produce the necessary amounts of light elements observed in second-generation stars (SG stars) - those with enhanced $\mathrm{He}, \mathrm{N}, \mathrm{Na}$, and $\mathrm{Al}$ abundances. This issue is referred to as the mass budget problem (Renzini 2008).

In this paper, we report the serendipitous discovery of a population of bulge field stars with abundance patterns that are similar to those found in stars from GCs. Characterized by high [N/Fe], which is correlated with $[\mathrm{Al} / \mathrm{Fe}]$ and anticorrelated with $[\mathrm{C} / \mathrm{Fe}]$, these stars are homogeneously distributed across the Galactic bulge and, to first order, are spatially and kinematically indistinguishable from the rest of the bulge field population. We characterize this new population and discuss the implications of this finding for our understanding of both bulge and GC formation.

In Section 2, the data employed in this paper are described. The results are presented in Section 3 and discussed in Section 4. Our conclusions are summarized in Section 5.

\section{DATA AND SAMPLE}

The results reported in this paper are based on elemental abundances for a large sample of Galactic stars from Data Release 12 (DR12; Alam et al. 2015) of the Apache Point Observatory Galactic Evolution Experiment (APOGEE; Majewski et al. 2016). One of four Sloan Digital Sky Survey-III (SDSS-III; Eisenstein et al. 2011) experiments, APOGEE used a new spectrograph on the Sloan $2.5 \mathrm{~m}$ telescope (Gunn et al. 2006) at APO to obtain high-quality $H$-band spectra $(R=22500, \mathrm{~S} / \mathrm{N} \sim 100$ per half-resolution element) for over 136000 stars distributed across all Galactic components, from which precision radial velocities, stellar parameters, and abundances for up to 15 elements have been obtained. Further in-depth information on the APOGEE survey, data, and the

\footnotetext{
${ }^{2}$ For reviews on star-to-star abundance variations in GCs, see Gratton, Sneden \& Carretta (2004) and Gratton et al. (2012).

3 The widespread use of the term 'generation' to refer to each of the multiple populations in GCs is associated with the sometimes tacit acceptance of a specific set of scenarios for their origin. Despite the fact that existing models do not account for the extant data in detail, which calls into question the physical reality of these scenarios, we choose to adopt the same nomenclature for consistency with current jargon.
}

data reduction pipeline can be found in Majewski et al. (2016), Holtzman et al. (2015), and Nidever et al. (2015), respectively. The APOGEE Stellar Parameters and Chemical Abundances Pipeline (ASPCAP) is described in detail in García Pérez et al. (2016). Heliocentric distances, $d_{\odot}$, were based on a Bayesian analysis of the stellar parameters, adopting as priors a history of star formation and IMF, and the PARSEC theoretical isochrones (Bressan et al. 2012). For more details on the method, see Binney et al. (2014). Possible systematic effects in our distances were assessed by employing the linear distance estimator from Schönrich, Binney \& Asplund (2012), with the conclusion that distances to the giants may be too long by about 25 per cent in general, with indications for possibly a larger systematic effect at $\log g<2$, which does not affect our results.

In this paper, we concern ourselves with a subset of the APOGEE sample, namely stars located in the Galactic bulge. Moreover, because we are interested in searching for stars with chemical signatures typical of GC members, we focus on the abundances of Fe, C, $\mathrm{N}$, and Al. With the above constraints in mind, the sample analysed in this study is defined by the following set of criteria:

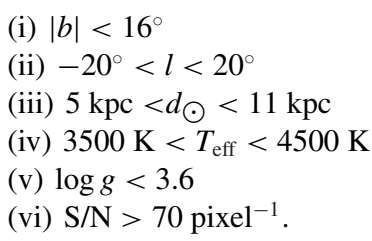

The final sample so selected amounts to a total of 5148 stars. Because we are interested in field stars only, suspected or known members of GCs located within the spatial region defined above were identified and excluded from the sample. A star was considered to be a GC member if it is located within its tidal radius, if its radial velocity differs from that of the GC (when available) by no more than $20 \mathrm{kms}^{-1}$, and if its metallicity differs from that of the cluster by no more than 0.3 dex. In this way, eight stars were identified as GC members, within the range of distances above, leaving us with a grand total of 5140 field stars. The surface gravity criterion is meant to avoid contamination of the sample by nearby dwarfs which are in any case extremely rare in APOGEE bulge pointings, given the shallower magnitude limit adopted in these fields - see Zasowski et al. (2013) for details. The $T_{\text {eff }}$ criterion is aimed at maximizing the overall quality of the abundances considered. At $T_{\text {eff }}<3500 \mathrm{~K}$, APOGEE does not presently provide elemental abundances, because the spectral library upon which ASPCAP is based does not extend to such low temperatures. At the other end of the $T_{\text {eff }}$ range, stars hotter than $4500 \mathrm{~K}$ are not considered because the abundances of $\mathrm{C}$ and $\mathrm{N}$ are uncertain in that $T_{\text {eff }}$ regime (see discussion in Mészáros et al. 2015). The uncertainty arises because ASPCAP determines these abundances from the strengths of $\mathrm{CN}$ and CO lines, which become too weak for $T_{\text {eff }}>4500 \mathrm{~K}$ at relatively low metallicities $([\mathrm{Fe} / \mathrm{H}] \lesssim-1)$. This sample is supplemented with data for stars belonging to various Galactic GCs targeted by APOGEE (for details, see Zasowski et al. 2013; Mészáros et al. 2015), which meet the selection criteria on stellar parameters and $\mathrm{S} / \mathrm{N}$ listed above. The latter data set is used to define the locus occupied by GC stars in chemical diagnostic plots.

The DR12 APOGEE abundances employed in this work are based on $\chi^{2}$-minimization of the observed spectra against a large spectral library calculated on the basis of state-of-the-art model photospheres (Mészáros et al. 2012) and a customized line list (Shetrone et al 2015). Specifically, synthetic spectra were calculated using the ASSET code (Koesterke 2009), using local thermodynamic 
equilibrium, plane-parallel, model photospheres calculated with the ATLAS9 code (Kurucz 1993). Giant stars with $T_{\text {eff }} \lesssim 4000 \mathrm{~K}$ and low surface gravity present extended atmospheres, which can invalidate the plane-parallel approximation. Sphericity effects cause a dilution of radiative flux that leads to lower temperatures in the upper layers of the photosphere (Plez, Brett \& Nordlund 1992), potentially affecting the strengths of molecular lines. To check for any important systematics coming from adoption of plane-parallel photospheres, DR12 abundances were compared with those obtained from a run of ASPCAP adopting a new spectral library (Zamora et al. 2015), calculated using the Turbospectrum spectrum synthesis code (Alvarez \& Plez 1998; Plez 2012) and the MARCS model atmospheres (Gustafsson et al. 2008), which adopt spherical symmetry for all models with $\log g \leq 3$. These comparisons showed that the elemental abundances relevant to this work are not affected by adoption of those more sophisticated analysis methods, so we proceed by adopting DR12 numbers for the remainder of this study.

A cautionary note is in order before proceeding with the analysis. The elemental abundances from APOGEE are subject to zeropoint differences relative to optical studies. Calibrations between the APOGEE and literature abundance scales were performed by Mészáros et al. (2013) on DR10 data (Ahn et al. 2014), whereas, for DR12, a similar procedure was followed, as described by Holtzman et al. (2015). In this study, unless otherwise noted, we opt to work with the uncorrected data, to take full advantage of the homogeneity and internal consistency of the ASPCAP-derived elemental abundances, which is crucial when comparing samples from different systems, such as the Galactic bulge and GCs. As discussed by Holtzman et al. (2015), the zero-point corrections are small and do not affect our conclusions in any important way.

All the data employed in this work are available in the form of electronic tables accessible at the 'Additional Supporting Information' section in the online version of this paper.

\section{RESULTS}

In this section, we describe the central finding reported in this paper, namely the discovery of stars in the field of the inner Galaxy that possess chemical compositions that are suggestive of a GC origin. In Sections 3.1 and 3.2, the behaviour of the sample in chemical composition diagnostic plots is characterized, and the identification of the newly discovered stellar population is described. Section 3.3 is aimed at reassuring the reader of the reality of the high nitrogen abundances resulting from ASPCAP. Sections 3.4 and 3.5 discuss the possible contamination of our sample by other stars that could potentially present the same abundance patterns, respectively intermediate-mass asymptotic giant branch (AGB) stars and the secondary remnants of mass-transfer binaries, concluding that such contaminations are minimal and not likely to affect our results importantly. The reader solely interested in the discussion of the main results may skip the latter three sub-sections.

\subsection{Distribution of field stars in the $[\mathrm{Fe} / \mathrm{H}]-[\mathrm{N} / \mathrm{Fe}]$ plane}

We start by examining the distribution of our sample stars in the $[\mathrm{Fe} / \mathrm{H}]$ versus $[\mathrm{N} / \mathrm{Fe}]$ plane, which is presented in Fig. 1(a). Two main features are worth noticing in this plot. First, it is clear that the relation between $[\mathrm{N} / \mathrm{Fe}]$ and $[\mathrm{Fe} / \mathrm{H}]$ of the sample is non-monotonic. The bulk of the stars with $[\mathrm{Fe} / \mathrm{H}] \gtrsim-0.7$ follow a clear correlation between $[\mathrm{Fe} / \mathrm{H}]$ and $[\mathrm{N} / \mathrm{Fe}]$. For lower metallicities, there is a reversal in that relation, such that $[\mathrm{N} / \mathrm{Fe}]$ actually decreases with increasing $[\mathrm{Fe} / \mathrm{H}]$. Secondly, a large number of stars, highlighted by

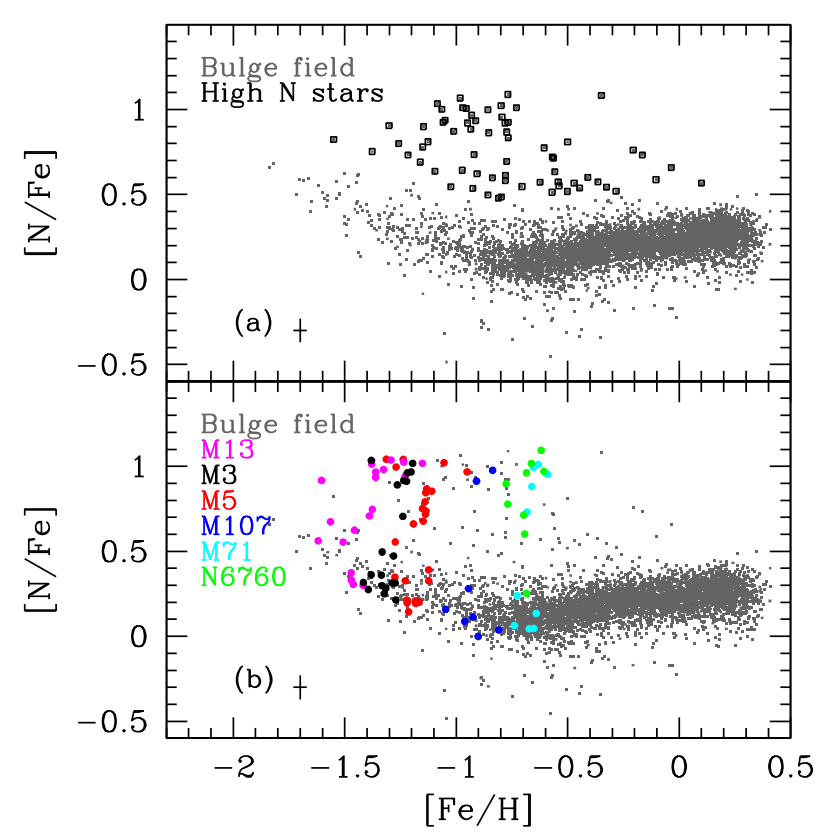

Figure 1. Distribution of the 5140 sample stars in the $[\mathrm{N} / \mathrm{Fe}]$ versus $[\mathrm{Fe} / \mathrm{H}]$ plane. (a) Shown with small grey dots are stars selected as described in Section 2. Squares indicate N-rich stars, defined as stars deviating by more than $4 \sigma$ from a sixth-order polynomial fit to the relation between $[\mathrm{N} / \mathrm{Fe}]$ and $[\mathrm{Fe} / \mathrm{H}]$. (b) The same field sample is compared to APOGEE data for Galactic GCs within a smaller range of metallicities. GC stars span the range of $[\mathrm{N} / \mathrm{Fe}]$ covered by field stars of same metallicity. FG GC stars are a good match to the $[\mathrm{N} / \mathrm{Fe}]$ versus $[\mathrm{Fe} / \mathrm{H}]$ relation of field stars, as expected (see, e.g., Gratton et al. 2012), with SG stars spanning larger values of [N/Fe] for fixed $[\mathrm{Fe} / \mathrm{H}]$, thus occupying the same locus as the N-rich stars reported in this paper. The sharp edge in the data distribution at $[\mathrm{N} / \mathrm{Fe}] \sim 1$ is an artefact of the $[\mathrm{N} / \mathrm{Fe}]$ upper limit in the ASPCAP spectral library.

adoption of larger symbols, are scattered above the main swath of data points at all metallicities, and there are a smaller number of outliers towards low [N/Fe] values. A total of 67 high-[N/Fe] outliers, highlighted by larger symbols, are identified by fitting a sixth-order polynomial to the $[\mathrm{N} / \mathrm{Fe}]$ versus $[\mathrm{Fe} / \mathrm{H}]$ relation and selecting stars that deviate from the fit by more than $4 \sigma$. For reasons that are explained in Section 3.2, we remove stars with $[\mathrm{C} / \mathrm{Fe}]>+0.15$, leaving a total sample of 58 stars, which we henceforth refer to as $N$-rich stars. They are listed in Table A1.

Post-main-sequence evolution complicates the interpretation of carbon and nitrogen abundances in giant stars (see, e.g., Lardo et al. 2012). The surface abundances of these elements are affected by the combined effects of the first dredge-up and extra mixing (for a thorough review, see Karakas \& Lattanzio 2014). The first dredge-up is a well-understood physical process that involves the deepening of the convection zone as the star evolves up the giant branch, causing material processed through the $\mathrm{CN}(\mathrm{O})$ cycle to be brought to the stellar surface, changing the atmospheric abundances of some elements. Extra mixing, on the other hand, is a non-convective process that brings about additional changes to atmospheric abundances in red giants (e.g. Gratton et al. 2000). The efficiency of extra mixing is a function of stellar metallicity (Martell, Smith \& Briley 2008), and the physical process responsible for it has not been established yet. Some of the ideas proposed involve stellar rotation, thermohaline mixing, magnetic fields, meridional circulation combined with turbulent diffusion, or perhaps some combination of some of these processes (e.g. Renzini \& Voli 1981; Charbonnel \& Lagarde 2010; Angelou et al. 2012; Karakas \& Lattanzio 2014). An in-depth 


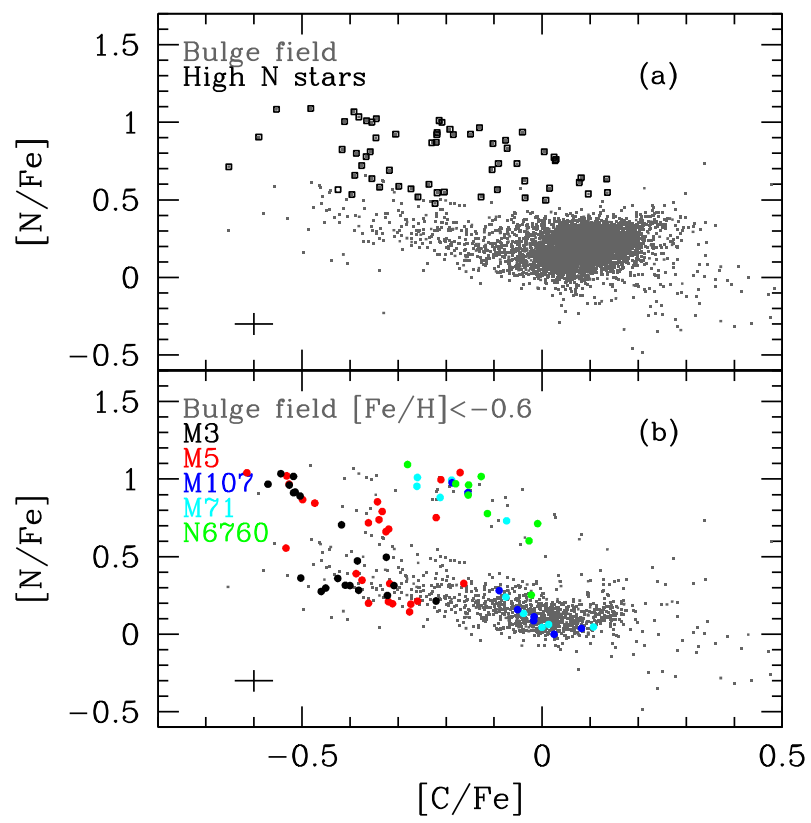

Figure 2. Stars from Fig. 1 in the $[\mathrm{C} / \mathrm{Fe}]$ versus $[\mathrm{N} / \mathrm{Fe}]$ plane. (a) This is the figure where a population of stars with high nitrogen abundance, anticorrelated with carbon (N-rich stars), was first identified. There potentially are two populations of $\mathrm{N}$-rich stars, with $[\mathrm{N} / \mathrm{Fe}]$ up to $\sim 1$, and another with lower $[\mathrm{N} / \mathrm{Fe}]$ and a smaller range of $[\mathrm{C} / \mathrm{Fe}]$ (roughly between -0.5 and -0.2). Stars marked as squares are the same as those in Fig. 1, except for those with $[\mathrm{C} / \mathrm{Fe}]>+0.15$, because those seem to depart from the anticorrelation between $\mathrm{C}$ and $\mathrm{N}$. Moreover, stars with such high $[\mathrm{C} / \mathrm{Fe}]$ are not usually found in Galactic GCs. (b) Bulge field stars are compared with members of Galactic GCs. The GC members are distributed across discrete 'branches' within which the two abundances are anticorrelated. Each branch belongs to a distinct GC stellar population, and the anticorrelation within each branch is due to stellar evolution. In each GC, branches with the lowest [N/Fe] abundances correspond to FG populations, whose abundance patterns are indistinguishable from the bulk of field stars of same metallicity. GC branches with higher [N/Fe] correspond to SG stars, which occupy the same locus in the C-N plan as N-rich stars, showing an identical anticorrelation between $[\mathrm{C} / \mathrm{Fe}]$ and $[\mathrm{N} / \mathrm{Fe}]$. Bulge field stars with $[\mathrm{Fe} / \mathrm{H}]<-0.6$ and M13 members are excluded, to keep the comparison to stars within the same metallicity range.

analysis of mixing is beyond the scope of this paper. While deep mixing hampers interpretation of these data in terms of the history of nitrogen and carbon enrichment of the Galaxy, it has no impact on our results, as discussed in Section 3.2.

\subsection{N-rich stars}

Now we turn our attention to the high-[N/Fe] outliers in Fig. 1(a). A diagnostic plot that can shed light on the nature of those stars is shown in Fig. 2(a) where $\mathrm{N}$-rich stars are again highlighted by large symbols. One can see that the $\mathrm{N}$-rich stars are distributed along at least two discrete bands where $[\mathrm{N} / \mathrm{Fe}]$ is anticorrelated with $[\mathrm{C} / \mathrm{Fe}]$. The most obvious of these branches contains the stars with highest $[\mathrm{N} / \mathrm{Fe}]$ in our sample, and runs roughly between $\{[\mathrm{C} / \mathrm{Fe}],[\mathrm{N} / \mathrm{Fe}]\}$ $=\{-0.5,+1.1\}$ and $\{0.0,+0.8\}$. There also seems to be a second branch of stars with more intermediate values of $[\mathrm{N} / \mathrm{Fe}]$, running approximately between $\{[\mathrm{C} / \mathrm{Fe}],[\mathrm{N} / \mathrm{Fe}]\}=\{-0.4,+0.8\}$ and $\{-0.1,+0.4\}$. This intermediate- $\mathrm{N}$ branch is less obvious and partly merged with the main body of the stars with $[\mathrm{C} / \mathrm{Fe}] \lesssim-0.1$. Nevertheless, close inspection of Fig. 2 reveals a fairly clear intermediate-
$\mathrm{N}$ sequence, which is well separated from the main body of $\mathrm{N}$-normal stars for $[\mathrm{C} / \mathrm{Fe}]<-0.2$.

These individual branches of anticorrelated carbon and nitrogen abundances strongly resemble those long known to exist in Galactic GCs (e.g. Norris et al. 1981; Hesser et al. 1982). As mentioned above, the mixing of the products of high-temperature shell hydrogen burning into the atmospheres of the giant branch stars leads to a large increase in the relative abundance of nitrogen along the giant branch, at the expense of a comparable (or smaller, depending on the cluster or data set) decrease in the relative abundance of carbon. Over time, red giant branch (RGB) stars evolve along these branches, upwards and to the left through the $[\mathrm{C} / \mathrm{Fe}]-[\mathrm{N} / \mathrm{Fe}]$ plane. The similarity with GC stars is further suggested by Fig. 2(b), where APOGEE data for stars belonging to a few Galactic GCs, spanning the same range of metallicities as the N-rich stars, are overlaid on the field sample. This plot indicates that the GC member stars follow similar $\mathrm{C}-\mathrm{N}$ anticorrelation sequences that run parallel to those observed in the field. In this figure, GCs show clear evidence for the presence of more than one $\mathrm{C}-\mathrm{N}$ anticorrelation sequence. Each of these sequences corresponds to one of the multiple populations commonly found in Galactic GCs (see Mészáros et al. 2015, for a discussion of the APOGEE data on these clusters).

Since the stars in both field and GC samples are red giants that have undergone first dredge-up, their nitrogen abundances have increased from their initial main-sequence (MS) values at the expense of ${ }^{12} \mathrm{C}$. Expected variations are a function of stellar mass and, to a lesser degree, metallicity, but $[\mathrm{N} / \mathrm{Fe}]$ typically increases by $\sim 0.3$ dex (e.g. Charbonnel \& Lagarde 2010), whereas [C/Fe] decreases by $\sim-0.15$. The stars belonging to the 'normal' sequence reach $[\mathrm{N} / \mathrm{Fe}]$ as high as $\sim+0.5$ and $[\mathrm{C} / \mathrm{Fe}]$ around $\sim-0.5$, which may be extreme values for first dredge-up in low-mass red giants, but can be accounted for by efficient deep mixing during the first-ascent giant branch. Taking the example of a star with $[\mathrm{Fe} / \mathrm{H}]=-1.0$ and the observed trend in Fig. 1, the expected initial ${ }^{14} \mathrm{~N}$ abundance would be $[\mathrm{N} / \mathrm{Fe}] \sim+0.1$, or $A(\mathrm{~N}) \sim 6.8$ for a solar nitrogen value of $\mathrm{A}_{\odot}=7.86$. At the same $[\mathrm{Fe} / \mathrm{H}]$, normal stars in our sample have an average value of $\sim-0.15$ for $[\mathrm{C} / \mathrm{Fe}]$, which corresponds to $A(\mathrm{C}) \sim 7.25$. If most of this initial carbon $\left({ }^{12} \mathrm{C}\right)$ is converted to ${ }^{14} \mathrm{~N}$ via the $\mathrm{CN}$ cycle, then the red giant nitrogen abundance could approach 7.4-7.5, or $[\mathrm{N} / \mathrm{Fe}]$ around +0.5 or +0.6 . Such values of ${ }^{14} \mathrm{~N}$ enhancements and ${ }^{12} \mathrm{C}$ depletions are observed in GCs, as seen in Figs 1 and 2.

The above simple $\mathrm{CN}$-cycle mixing scenario cannot account for the more extreme abundances in our sample. Most models proposed to account for the existence of such stars in GCs contend that they result from chemical evolution within the GCs themselves, as discussed in Section 1. In apparent support to that scenario, Fig. 2(b) indicates that the GC sequences at high $[\mathrm{N} / \mathrm{Fe}]$ tend to have, on average, lower $[\mathrm{C} / \mathrm{Fe}]$ than those at normal $[\mathrm{N} / \mathrm{Fe}]$, which is a manifestation of the well-documented fact that SG stars are both enhanced in $\mathrm{N}$ and diminished in $\mathrm{C}$ relative to FGs. Yet, the sample of high-[N/Fe] outliers in Fig. 1 contains stars with $[\mathrm{C} / \mathrm{Fe}]$ as high as $\sim+0.3$. Because such stars are not typically found in GCs, and because we want to avoid contamination by objects such as $\mathrm{CH}$ stars (e.g. McClure \& Woodsworth 1990; Karinkuzhi \& Goswami 2015), we restrict our sample to stars with $[\mathrm{C} / \mathrm{Fe}]<+0.15$, leaving us with a total of $58 \mathrm{~N}$-rich stars. This additional selection criterion has no impact on the conclusions presented in this paper.

Fig. 1(b) displays field and GC star data together on the [N/Fe] versus $[\mathrm{Fe} / \mathrm{H}]$ plane. The metal-poor GC M13 is included in this plot, so that GCs span as wide a metallicity range as possible, for a fair comparison with the field sample. It is clear that the GC 
population spans a wide range of $[\mathrm{N} / \mathrm{Fe}]$ at fixed $[\mathrm{Fe} / \mathrm{H}]$, due to a combination of deep mixing and stellar population complexity. For each GC, the data are distributed along discrete sets of data points, which are separated from each other by several tenths of a dex in $[\mathrm{N} / \mathrm{Fe}]$. The lowest discrete set, which is the one best sampled, possesses a total (i.e. 'peak to peak') scatter of $\sim 0.3$ dex in $[\mathrm{N} / \mathrm{Fe}]$. The individual groups correspond to each of the multiple stellar populations present in the GCs, whereas the $[\mathrm{N} / \mathrm{Fe}]$ scatter within each group is due to mixing along the giant branch (for more details, see Smith \& Martell 2003; Mészáros et al. 2015). Interestingly, the minimum $[\mathrm{N} / \mathrm{Fe}]$ for $\mathrm{GC}$ stars matches approximately the values for the field population at same $[\mathrm{Fe} / \mathrm{H}]$. In other words, the $[\mathrm{N} / \mathrm{Fe}]$ versus $[\mathrm{Fe} / \mathrm{H}]$ relation of the field population lies along the lower envelope of the $[\mathrm{N} / \mathrm{Fe}]$ distribution in the GC samples. This result is consistent with findings by other studies, which have characterized FG stars in GCs as having the same chemistry as field stars of same metallicity (e.g. Carretta et al. 2009). The scatter of the field population in $[\mathrm{N} / \mathrm{Fe}]$ is similar to that of the GC populations with lowest $[\mathrm{N} / \mathrm{Fe}]$ (FG stars), suggesting that the thickness of the stellar sequences at constant $[\mathrm{Fe} / \mathrm{H}]$ is due to deep mixing, at least in the low-metallicity regime.

The similarity between N-rich stars and SG stars from GCs can be further tested through examination of other abundance (anti)correlations typically found in GCs. The most popular is the anticorrelation between the abundances of $\mathrm{Na}$ and $\mathrm{O}$, but unfortunately sodium abundances in DR12 rely on an $\mathrm{Na}$ I line at $\lambda 1639.333 \mathrm{~nm}$ that is too weak in the spectra of stars with the typical $T_{\text {eff }}$ and metallicity of the N-rich sample for reliable abundances to be derived by ASPCAP. Examination of spectral fits for a handful of cool stars in the metal-rich end of our sample suggests the sodium abundances to be very high in N-rich stars and correspondingly lower in N-normal stars. Further work will be required to confirm this tentative result.

Aluminium is another element known to present strong variations in Galactic GCs (e.g. Kraft et al. 1997; Gratton et al. 2004, 2012), whose abundances should in principle be correlated with those of nitrogen and anticorrelated with those of carbon. Fortunately, Al lines are present in the APOGEE spectral region, so that ASPCAP abundances are reliable throughout the range of stellar parameters. Fig. 3(a) displays the same data and symbols plotted in Fig. 2(a) in the $[\mathrm{Al} / \mathrm{Fe}]-[\mathrm{N} / \mathrm{Fe}]$ plane. The bulk of the field population, located at $[\mathrm{N} / \mathrm{Fe}] \lesssim+0.5$, shows a slight anticorrelation between the two abundance ratios, whereas the $\mathrm{N}$-rich stars display a positive correlation. These trends can be understood by examination of Fig. 3(b), where a sub-sample including only stars with $[\mathrm{Fe} / \mathrm{H}]<-0.6$ is compared with APOGEE data for GCs in the same metallicity range. The SG stars in GCs occupy the same locus as N-rich stars, reinforcing the similarity between the two populations. Moreover, FG stars from GCs occupy the same locus as the general bulge field, displaying a similar anticorrelation between the two abundance ratios.

\subsection{Spectra and spectral fits}

Elemental abundances are the foundation upon which the results presented and discussed in this paper are built, so it is fitting that we provide evidence in support of the numbers reported by ASPCAP. A brief examination of typical APOGEE spectra for N-rich and $\mathrm{N}$-normal stars reassures us of the existence of a real chemical peculiarity in the former. Spectral comparisons are presented in Figs 4(a)-(c), where the spectra of an N-rich and a normal star are compared in relevant wavelength intervals.

Nitrogen abundances in APOGEE spectra are determined solely from the strengths of $\mathrm{CN}$ lines, which in turn are sensitive to other

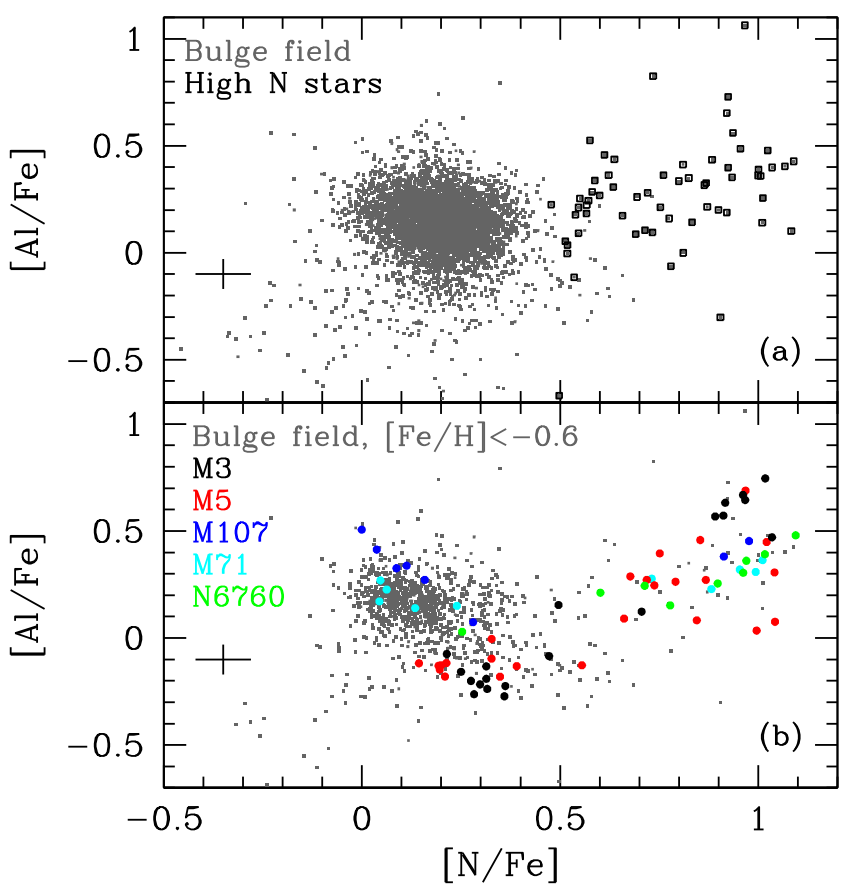

Figure 3. Sample stars in another chemical abundance diagnostic plot, adopting same symbols as in Figs 2(a) and (b). (a) A correlation between $[\mathrm{N} / \mathrm{Fe}]$ and $[\mathrm{Al} / \mathrm{Fe}]$ is evident in the N-rich sample (squares), whereas an anticorrelation appears to be present for stars with normal nitrogen abundances ([N/Fe] $\lesssim+0.5)$. (b) The SG stars in GCs, with $[\mathrm{N} / \mathrm{Fe}] \gtrsim+0.5$, occupy the same locus as the N-rich population, following a similar correlation between the two abundance ratios. On the other hand, FG stars in GCs follow the same trend as the lower [N/Fe] field stars. This plot corroborates the notion that the N-rich stars inhabit the same region of chemical composition space as SG stars from GCs. The GC sample has few counterparts to the field stars with intermediate nitrogen $(0.5 \lesssim[\mathrm{N} / \mathrm{Fe}] \lesssim 0.7)$ in panel (a). It is unclear whether this is a real effect or whether it is due to limitations in the APOGEE GC sample, which is relatively small.

parameters, chiefly $T_{\text {eff }}, \log g$, and the abundances of carbon and oxygen. The dependence of $\mathrm{CN}$ line strength on $T_{\text {eff }}$ and $\log g$ is due to a combination of the well-documented impact of temperature and pressure on molecular dissociation equilibrium (e.g. Russell 1934; Tsuji 1973) and the ratio between continuum and molecular line opacity (e.g. Bell \& Tripicco 1991). The abundances of C and $\mathrm{O}$ affect $\mathrm{CN}$ lines due to their impact on the concentration of the $\mathrm{CN}$ molecule in the stellar atmosphere, via molecular dissociation equilibrium (e.g. Russell 1934; Tsuji 1973). To simplify matters, we control for these parameters, by choosing two stars with nearly identical $T_{\text {eff }}, \log g,[\mathrm{C} / \mathrm{Fe}]$, and [O/Fe], but vastly different [N/Fe], for our comparison. We stress that the 'normal' comparison star is chosen not to be a representative of an FG counterpart to the $\mathrm{N}$-rich star, but rather to have stellar parameters and abundances that are identical to that of the N-rich stars, so as to highlight the impact of nitrogen abundance variations in the stellar spectrum. The stars selected are 2M17480576-2445000 (N-rich) and 2M18081125-2426140 (N-normal); their stellar parameters and chemical abundances are displayed in Fig. 4(a). The spectra of these two stars are compared in a wavelength range containing several CO lines (Mészáros et al. 2015), some of which are indicated by vertical tick marks. One can immediately conclude that the CO lines are similar between the two spectra. In fact, the N-normal star has slightly stronger CO lines, which is possibly due to a combination of a slightly higher carbon abundance and slightly lower surface gravity. The strong bandhead at $\lambda \sim 1619 \mathrm{~nm}$ shows a particularly larger difference, which is 


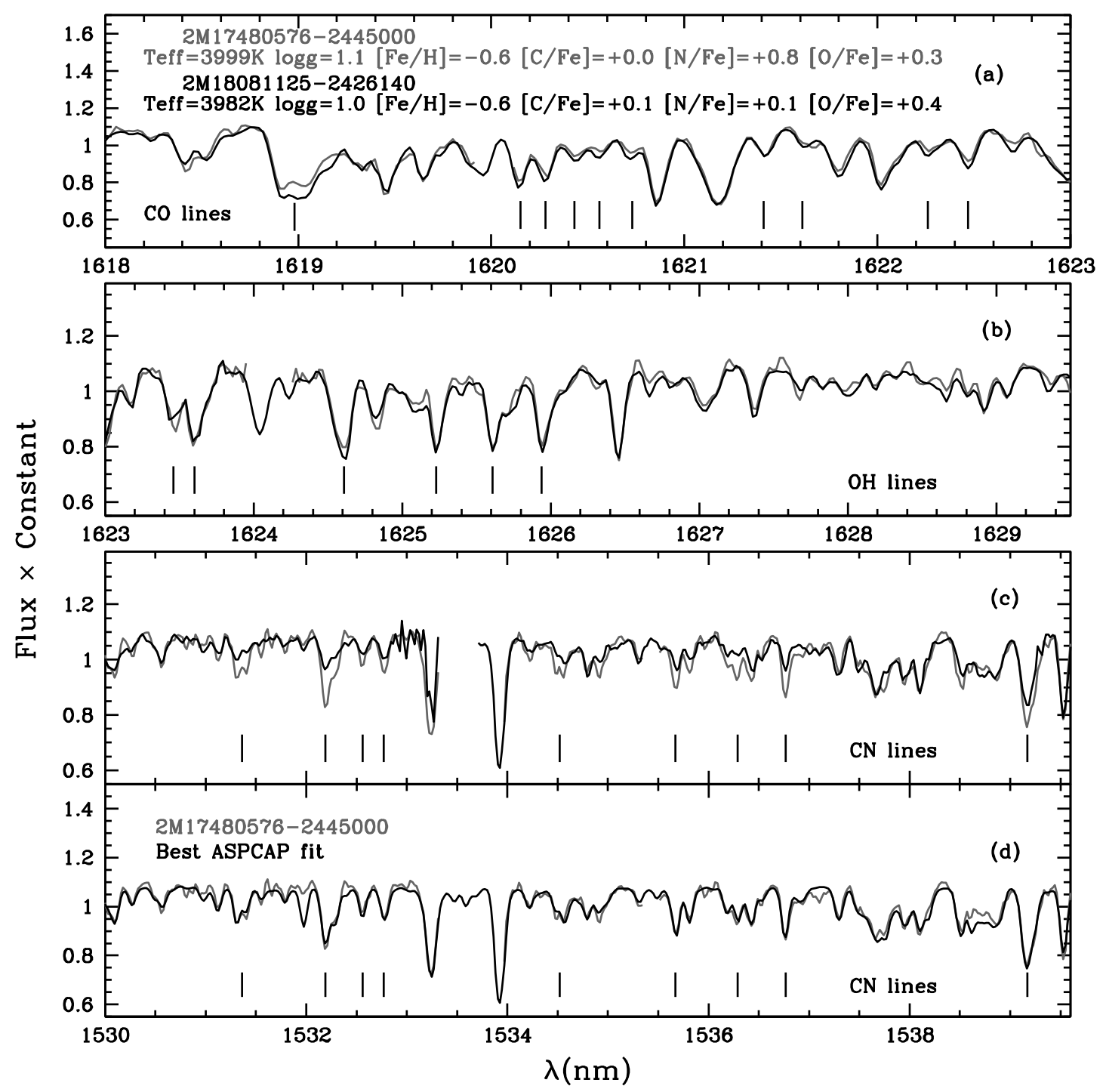

Figure 4. APOGEE spectra and spectral fits. (a) Comparison between the spectra of a normal (black) and an N-rich star (grey), with similar stellar parameters and similar abundances of carbon and oxygen, as labelled. The vertical ticks indicate the positions of CO lines, which are similar in the spectra of the two stars, with the exception of the lines at $\sim 1619 \mathrm{~nm}$, which are contaminated by atomic lines (see the text). (b) Spectra of the same stars, now in a region containing $\mathrm{OH}$ lines, which are indicated by tick marks. OH lines are also similar in the spectra of the two stars. (c) Same spectra in a wavelength range containing CN lines, indicated by vertical tick marks. The N-rich star has much stronger $\mathrm{CN}$ lines. Given the similarity in stellar parameters and the strengths of $\mathrm{CO}$ and $\mathrm{OH}$ lines, the difference in $\mathrm{CN}$ lines can only be due to the N-rich star indeed having a much higher abundance of nitrogen. (d) The spectrum of the N-rich star from panel (c) is compared to the best fit from ASPCAP. The match is excellent, which lends confidence to the quality of nitrogen abundances delivered by ASPCAP. The match to the spectrum of the N-normal star (not shown) is equally good. Bad detector pixels, as well as those characterized by strong airglow residuals, are masked.

possibly due to contamination by lines due to $\mathrm{Si}, \mathrm{V}$, and $\mathrm{Sc}$. In Fig. 4(b), spectra for the same stars are now compared in a region containing several $\mathrm{OH}$ lines and, again, line strengths are similar in the spectra of the two stars. The combination of these two empirical results, in view of the fact that the two stars have nearly the same atmospheric parameters, means that they must have similar abundances of carbon and oxygen, as indicated by the ASPCAP results. We now turn to Fig. 4(c), where the spectra are compared in a region containing $\mathrm{CN}$ lines, again indicated by vertical tick marks. The $\mathrm{N}$-rich star has remarkably stronger $\mathrm{CN}$ lines which, in view of the similarity between the two stars in all the other relevant parameters, can only mean that it has much higher nitrogen abundance. ASPCAP tells us that $[\mathrm{N} / \mathrm{Fe}]$ in the $\mathrm{N}$-rich star is higher than in the N-normal star by 0.7 dex. To first order, the quality of the ASPCAP result is verified by comparison between the observed spectrum and the best ASPCAP fit (Fig. 4d), where it can be seen that the CN lines (and indeed most of the spectrum) are well reproduced. The quality of the ASPCAP fits to the spectra of N-normal stars can be verified in other APOGEE publications (e.g. Holtzman et al. 2015; García Pérez et al. 2016). We conclude that the nitrogen abundance differences, which are the basis for our identification of a new stellar population in the inner Galaxy, are highly reliable.

\subsection{Evolutionary stage of the N-rich stars}

Post-main-sequence evolution is known to affect the surface abundances of giant stars during the RGB and AGB evolutionary stages, in ways that resemble those observed in the N-rich sample (e.g. 


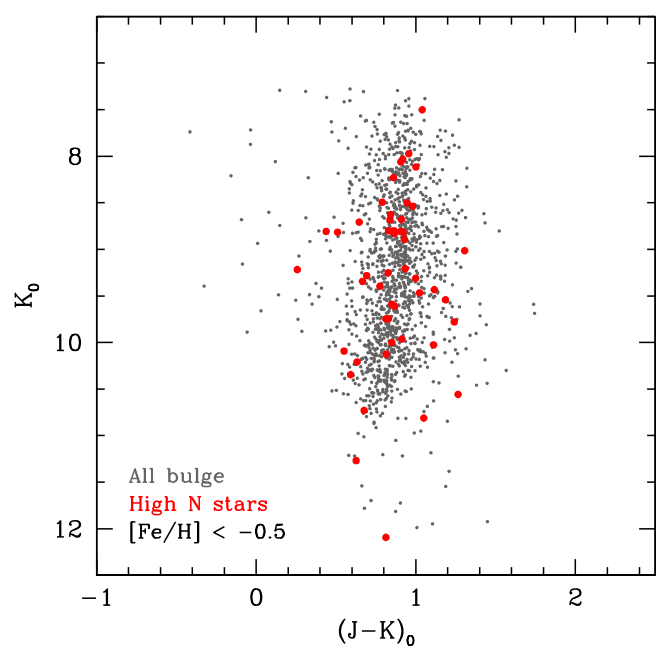

Figure 5. The dereddened 2MASS CMD for the sample studied in this paper. 'Normal' stars are plotted using small grey symbols, and N-rich stars with larger red symbols. Only stars with $[\mathrm{Fe} / \mathrm{H}]<-0.5$ are shown for both samples, to minimize effects due to differences between the metallicity distributions of the two samples (the normal stars extend to much higher metallicities; see Section 3.6). There are no noticeable differences between $\mathrm{N}$-rich and $\mathrm{N}$-normal stars, suggesting that the $\mathrm{N}$-rich stars are unlikely to be dominated by AGB stars.

Renzini \& Voli 1981; Charbonnel \& Lagarde 2010). In particular, the abundance pattern identified in our sample of N-rich stars is characteristic of the surfaces of intermediate-mass $\left(3-4 \mathrm{M}_{\odot}\right)$ AGB stars that have undergone hot bottom burning. The presence of such young, moderately metal-poor stellar populations in the Galactic bulge would have important implications. Therefore, the interpretation of our results depends crucially on establishing the evolutionary stage of the stars under analysis. The large luminosities and low temperatures of AGB stars, combined with the relatively bright APOGEE magnitude limits in bulge fields (Zasowski et al. 2013) and the focus of our sample on $T_{\text {eff }}<4500 \mathrm{~K}$, may bias our sample towards a high fraction of AGB stars. The possible existence of such a bias is examined in this section.

\subsubsection{Known AGB stars and colour-magnitude diagram}

We start by searching for known candidate AGB stars in the N-rich sample. Inspection of the IDs of AGB stars targeted ${ }^{4}$ in the Galactic Centre field (field ID 'GALCEN' in the APOGEE data base) showed that none of them is included in the N-rich sample - in fact, they are almost all too cool $\left(T_{\text {eff }}<3500 \mathrm{~K}\right)$ for ASPCAP to deliver reliable abundances. The only exception is star 2M17451937-2914052, for which ASPCAP finds $T_{\text {eff }}=3690 \pm 91 \mathrm{~K},[\mathrm{Fe} / \mathrm{H}]=-0.68 \pm$ $0.04,[\mathrm{C} / \mathrm{Fe}]=+0.54 \pm 0.05,[\mathrm{~N} / \mathrm{Fe}]=+0.16 \pm 0.08,[\mathrm{Al} / \mathrm{Fe}]=$ $-0.10 \pm 0.10$, which places this star clearly outside the chemical composition locus occupied by N-rich stars.

The next obvious way of checking for the presence of an AGB bias is by comparing the distribution of $\mathrm{N}$-rich and $\mathrm{N}$-normal stars in the colour-magnitude diagram (CMD). Fig. 5 displays the N-rich and $\mathrm{N}$-normal samples in the dereddened 2MASS (Skrutskie et al. 2006)

\footnotetext{
${ }^{4}$ AGB star candidates were selected from the sample of Schultheis et al. (2003), which is based on $\mathrm{H}_{2} \mathrm{O}$ and $\mathrm{CO}$ absorption, ISOGAL mid-IR excesses, and light curves. We refer the reader to that paper for further details.
}

CMD. Dereddening was performed using $A_{K}$ from DR 12 (adopting the A_K_TARG parameter), which was inferred through the RJCE method (Majewski, Zasowski \& Nidever 2011), and adopting the extinction law from Indebetouw et al. (2005). The sample plotted is limited to $[\mathrm{Fe} / \mathrm{H}]<-0.5$, to minimize differences between $\mathrm{N}$-normal and $\mathrm{N}$-rich samples that are purely due to differences in their metallicity distributions (see discussion in Section 3.6). One can immediately notice that the two sub-samples occupy the same locus in the CMD, suggesting that the $\mathrm{N}$-rich sample is not biased towards AGB stars relative to the remainder of the field sample. In other words, the AGB/RGB ratio of the N-rich sample is likely to be the same as that of the rest of the field sample. The same conclusion is drawn from comparison of the two samples in a (reddening and distance independent) $T_{\text {eff }}$ versus $\log g$ diagram (not shown). A more quantitative assessment can be made by selecting $\mathrm{N}$-rich and $\mathrm{N}$-normal stars within a narrow range of metallicities and compare the differences in colour between the two samples for the same magnitude, where AGB and RGB stars can differ in $J-K$ by as much as 0.05 mag (e.g. Girardi et al. 2000). We proceeded by fitting fiducials to the two samples in the CMD and comparing the colours of the fiducials for a given magnitude. For instance, at $H=$ 9, we obtain $J-K=0.91 \pm 0.04$ and $0.91 \pm 0.06$ for N-rich and N-normal, respectively, and at $H=10$ we obtain $J-K=0.85$ for both samples, with same uncertainties. In conclusion, we find no difference between $\mathrm{N}$-rich stars and the rest of the field sample in the dereddened 2MASS CMD, which is consistent with no substantial difference in AGB contribution to the N-rich sample and the rest of the field.

\subsubsection{Infrared excess}

The presence of AGB stars in our sample can be further assessed by detection of photometric signatures of the presence of circumstellar dust. Vigorous mass-loss during late stages of AGB evolution is responsible for the formation of dusty envelopes (e.g. Habing 1996), which manifest themselves through excess radiation at long wavelengths (e.g. García-Lario et al. 1997). In Fig. 6, N-normal and N-rich stars are shown in a 2MASS dereddened colour-colour diagram. The loci occupied by RGB and MS stars, AGB stars, young stellar objects (YSOs), and planetary nebulae, according to the study by García-Lario et al. (1997), are separated by lines and indicated by labels. ${ }^{5}$ Because of the presence of circumstellar dust, AGB stars occupy a locus towards colours redder than those of RGB and MS stars in this diagram. From the distribution of the data points, one can see that the vast majority of the sample is located in the RGB+MS sequence, with only about 1 per cent of all the stars positioned in the AGB area of the plot. In particular, all N-rich stars inhabit the RGB+MS part of the diagram, which provides further evidence for the absence of an important contribution of AGB stars to our N-rich sample.

Fig. 7 shows our sample stars on colour-magnitude and colourcolour diagrams based on Spitzer IRAC dereddened photometry (again using the extinction law by Indebetouw et al. 2005). Mid-IR colours are particularly sensitive to the presence of dust, so these

\footnotetext{
${ }^{5}$ The loci of the various object types in the colour-colour diagram were defined by García-Lario et al. (1997) on the basis of photometry on the Koornneef system (Koornneef 1983). Considering small zero-point differences between different photometric systems and an error of 5-10 per cent in photometry of bright stars, the mismatch between the loci in the two systems should be at most $0.1 \mathrm{mag}$, which does not affect our conclusions.
} 


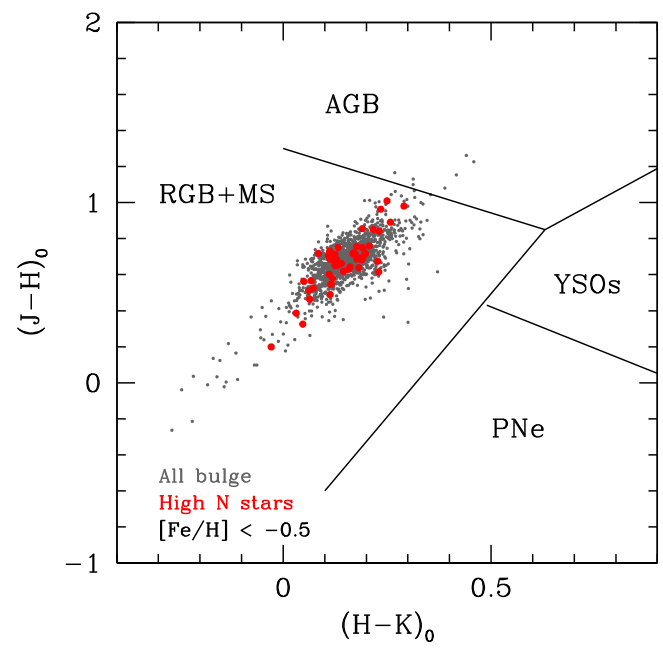

Figure 6. Sample stars in the 2MASS dereddened colour-colour plot, adopting same symbols as Fig. 5. The colour-colour plane is divided into sections populated by various types of NIR-bright objects, following the study by García-Lario et al. (1997), as indicated by the labels. The vast majority of the sample occupies the RGB+MS region of the diagram, which shows that there are very few dusty AGBs in the sample. In particular, none of the $\mathrm{N}$-rich stars occupies the AGB part of the diagram.

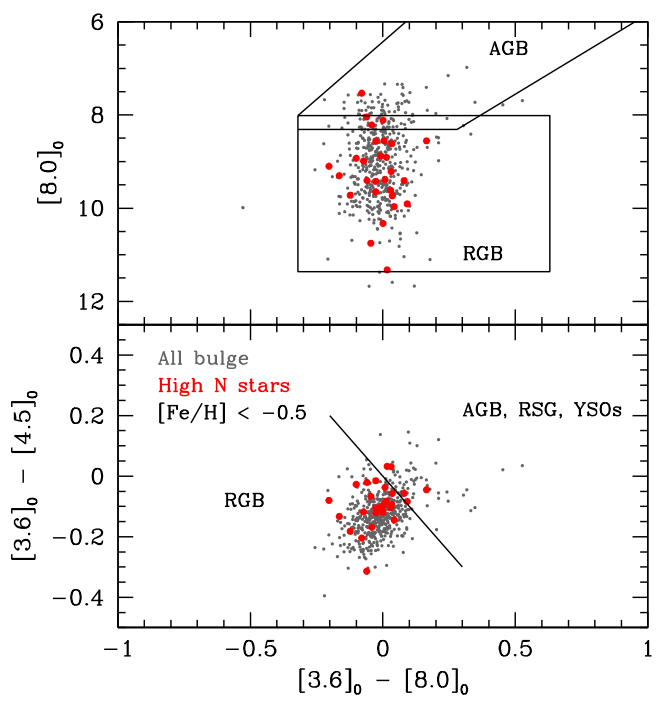

Figure 7. Using dereddened Spitzer IRAC photometry to determine the evolutionary stage of our sample. Top panel: the loci of N-normal and $\mathrm{N}$ rich stars in the CMD are displayed. The areas of the diagram occupied by AGB and RGB stars are shown, suggesting that both the N-normal and $\mathrm{N}$-rich samples are dominated by RGB stars. Bottom panel: same sample as in the top panel, now in a colour-colour diagram. The solid line shows the frontier between the loci of RGB and other stellar types. Again, the evidence suggests that our sample is overwhelmingly dominated by RGB stars.

diagrams help spotting AGB stars with dusty envelopes. In the top panel, N-normal and N-rich stars are contrasted on a CMD, where the loci of RGB and AGB stars at a distance of $8 \mathrm{kpc}$, according to Ramírez et al. (2008), are indicated. As in the previous cases, the data strongly suggest a very small contribution by AGB stars to our sample. In the bottom panel, stars are displayed on an IRAC dereddened colour-colour diagram, where a line separates the locus of RGB from that of redder YSOs, red supergiants, and AGB stars. The loci were established from visual inspection of fig. 1 (bottom panel) of Marengo et al. (2007), who studied IRAC photometry for a sample of Galactic AGB stars. Again in this case, the vast majority of our sample stars are located safely outside the AGB region of the diagram, and within the locus commonly occupied by RGB stars.

\subsubsection{Variability}

AGB stars undergo thermal pulsations (e.g. Iben \& Renzini 1983), which manifest themselves observationally in the form of moderateto high-amplitude, long-period magnitude variations. Stellar types commonly associated with the AGB phase are Mira-type variables, long-period variables, semiregular variables, and the heavily obscured OH/IR stars (e.g. Jiménez-Esteban et al. 2006; Gray \& Corbally 2009).

To establish the occurrence of variability, one ideally needs multiepoch observations, preferably performed within the same photometric system, to minimize confusion due to zero-point differences. These are difficult to obtain because of the long periods associated with these variations, typically of the order of $10^{2}-10^{3} \mathrm{~d}$ (Gray \& Corbally 2009). In light of these requirements, probably the best source for photometric variability information on our sample stars would be long-term monitoring photometric surveys over large areas of the Galactic bulge, such as OGLE ${ }^{6}$ in the optical, and $\mathrm{VVV}^{7}$ survey in the NIR. Unfortunately, the overlap between the APOGEE and OGLE footprints is very small, so that only four N-rich stars were observed by OGLE. None of these stars are included in the OGLE catalogues of long- or double-period variables. The overlap with the VVV footprint is much larger, but due to the relatively bright limit of the APOGEE bulge sample $(H<11.2)$, saturation is a problem for VVV data (see, e.g., Catelan et al. 2014, for details).

We first examine magnitude differences between the two epochs included in the USNO-B catalogue (Monet et al. 2003), which contains 4296 stars in common with our bulge sample, of which 55 are N-rich stars. The data are shown in the top panel of Fig. 8, where $R$-band magnitude variations are plotted as a function of first-epoch magnitudes. The thin lines indicate $2 \sigma$ departures from the mean difference (thick line), where $\sigma$ is the photometric precision $(\sim 0.5 \mathrm{mag})$. Most stars are consistent with no variability. About 11 per cent of the N-rich stars varied by more than twice the photometric precision, whereas when the entire sample is considered, 13 per cent of the stars varied by more than $2 \sigma$. We find, for the whole sample, $\langle\Delta R 1\rangle=-0.4 \pm 0.9$ and for N-rich stars we find $\langle\Delta R 1\rangle=-0.4 \pm 1.0$. Combined, these numbers suggest that the N-rich stars do not include a higher fraction of variable stars than the rest of the sample. This result is insensitive to assumptions on the photometric precision of the USNO-B catalogue. Moreover, comparison of DENIS ${ }^{8}$ and 2 MASS data (below) suggests in fact that the variability information as inferred from this analysis of USNO data is actually questionable.

Identification of variable stars in the USNO-B catalogue is made difficult by its relatively low photometric precision. Therefore, we investigate more precise photometry from NIR catalogues, which

\footnotetext{
${ }^{6}$ Optical Gravitational Lensing Experiment (Udalski, Kubiak \& Szymanski 1997; Soszynski et al. 2013).

${ }^{7}$ Vista Variables in the Via Lactea (Saito et al. 2012; Catelan et al. 2014).

${ }^{8}$ DENIS is the Deep Near Infrared Survey of the Southern Sky (Epchtein et al. 1997).
} 

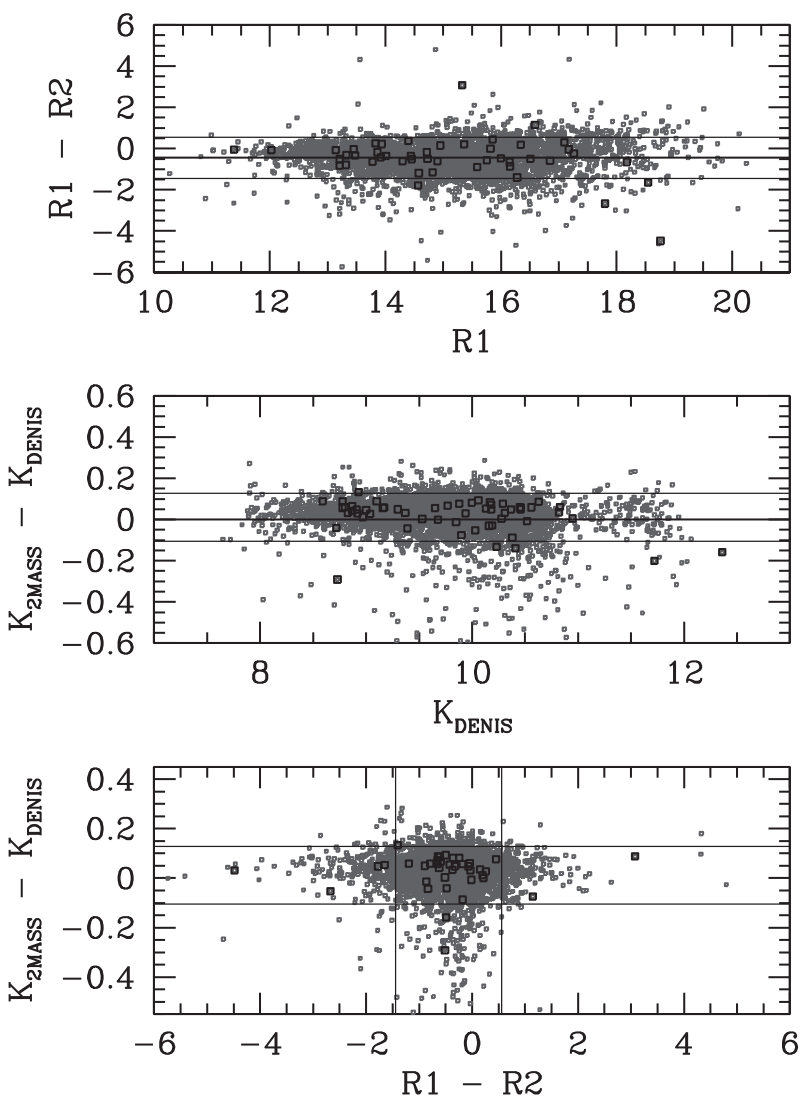

Figure 8. Assessment of the amount of variability in the N-rich sample. Top panel: variation in $R$ magnitude between two epochs in the USNO-B catalogue, as a function of magnitude in the first epoch. Small grey and solid black dots denote normal and N-rich stars, respectively. The thick horizontal line marks the position of the mean difference, and the two thin lines mark $\pm 2 \sigma$ departures from the mean, where $\sigma$ is the photometric precision. The mean difference and standard deviation are the same for the $\mathrm{N}$-rich and normal sample. Moreover, about 90 per cent of the points in both samples are within $2 \sigma$ of the mean, thus having no evidence for variability. Middle panel: same as above, for the difference between $K$-band magnitudes in 2MASS and DENIS, which have negligible zero-point differences. Conclusions are the same as in the top panel. Bottom panel: comparison between magnitude variations in optical and NIR from panels above. The thin lines again indicate $2 \sigma$ off the mean values, where $\sigma$ is again photometric precision. There is no correlation between magnitude variations. Stars for which there is an indication of variability in one band are consistent with no variation in the other band, suggesting that photometric errors may be responsible for strong variations observed.

also have well-determined photometric zero-points. Comparing 2MASS (Skrutskie et al. 2006) with UKIDSS $^{9}$ data, we found that $36 \mathrm{~N}$-rich stars are present in both catalogues out of a total common sample of 360 stars. Unfortunately, saturation of UKIDSS data is a problem for stars brighter than $K_{2 \mathrm{MASS}} \sim 10.5$, but the few stars fainter than that limit show no evidence for variations between the two catalogues.

We next examine possible variations between DENIS and 2MASS magnitudes, noting that Cabrera-Lavers \& Garzón (2003) showed zero-point differences between the two photometric systems to be not significant. A total of 4692 stars from our

${ }^{9}$ UKIRT Infrared Deep Sky Survey (Lawrence et al. 2007). sample are included in both catalogues, with 58 of them being N-rich. In Fig. 8, middle panel, the difference between $K$-band magnitudes in the 2MASS and DENIS systems is plotted as a function magnitude. Symbols and horizontal lines have the same meaning as in the top panel, adopting a photometric precision of 0.03 and $0.05 \mathrm{mag}$ for 2MASS and DENIS, respectively (Cioni et al. 2000; Skrutskie et al. 2006). The result of this comparison is consistent with what we found from analysis of the USNO-B data, with the vast majority of the stars being consistent with no variation. About $\sim 15$ per cent of the stars in the sample have variations larger than $2 \sigma$, and in the $\mathrm{N}$-rich sample, the number is $\sim 12$ per cent.

It is possible that some of the stars below the variability detection threshold of this comparison are small-amplitude red giant variables, such as those identified in the OGLE survey (Wray, Eyer \& Paczyński 2004; Soszyński et al. 2013). This in fact may partly explain why the scatter in the residuals $(\sim 0.1-0.14 \mathrm{mag})$ is larger than expected on the basis of photometric errors alone ( $\sim 0.06 \mathrm{mag})$. Most importantly, if the incidence of AGB stars was higher in the $\mathrm{N}$-rich sample, we would expect magnitude differences for those stars to present a larger scatter than for the rest of the sample. However, in agreement with USNO-B data, this is not what we find. In fact, the standard deviation is slightly smaller in the N-rich sample $(\langle\Delta K\rangle=0.01 \pm 0.11)$ than in the rest of the sample $(\langle\Delta K\rangle=0.01$ $\pm 0.14)$, which taken at face value suggests that the incidence of variable stars in the $\mathrm{N}$-rich sample is, if anything, smaller than in the rest of the sample.

In conclusion, analysis of two-epoch observations in both the optical and NIR consistently indicates that the fraction of variable stars in our sample is of the order of $\sim 10$ per cent. However, interestingly, the bottom panel of Fig. 8 shows that there is no correlation between magnitude variations in the USNO and 2MASS/DENIS catalogues, particularly in the N-rich sample. It is noteworthy that the stars for which variation is largest in one band in the other band fall within the area of the graph consistent with no variation. In other words, they are confined to the cross-shaped locus defined by the $\pm 2 \sigma$ limits in both bands. In short, stars that look variable in NIR do not in optical, and vice versa. This may be partly due to the fact that observations were collected at different epochs, but in this case one would expect that at least some of the largest deviants would fall outside the cross-shaped locus. Of relevance to this discussion is the fact that magnitude errors for the most deviant points in the USNOB sample are particularly large, even though they do not entirely account for the discrepancies. All in all, the bottom panel of Fig. 8 suggests that the fraction of variables in the N-rich sample may be indeed smaller than suggested by USNO and 2MASS-DENIS data considered in isolation.

We conclude that there is little evidence for a large contribution of AGB stars to our sample. Considering uncertainties in photometry and in the locus occupied by the various stellar types in the 2MASS and IRAC CMDs and colour-colour plots, it seems safe to conclude that our sample stars are predominantly composed of first-ascent RGB stars, with at most a small contribution by AGB stars. It is difficult to provide a solid estimate of the ratio between the number of AGB and RGB stars in our sample, without a more detailed analysis of the AGB candidates. Examination of the data presented in this section suggests that the ratio is probably no larger than $\sim 5-10$ per cent, being thus consistent with data from other old metal-poor stellar populations (see, e.g., Girardi et al. 2010, for a discussion). This topic will be the subject of further investigation in a future publication (Zamora et al., in preparation). 


\subsection{Incidence of mass-transfer binaries in N-rich sample}

Stars with $\mathrm{N}$ and $\mathrm{Al}$ overabundances can also originate through a binary mass-transfer channel. Intermediate-mass stars $\left(M \sim 3-8 \mathrm{M}_{\odot}\right)$ undergo hot bottom burning during their AGB phase, producing large amounts of $\mathrm{N}$ and $\mathrm{Al}$ (e.g. Ventura et al. 2013). When these objects are members of a binary with an appropriate semi-axis size, mass transfer takes place and the low-mass companion atmosphere is enriched with the products of AGB nucleosynthesis. The donor star evolves away from the AGB phase and eventually becomes a faint white dwarf, while the companion retains their chemical signature.

Establishing the presence of radial velocity variations among the $\mathrm{N}$-rich stars would be the most natural course of action towards estimating the fraction of such objects formed through the binary channel. However, most of the objects in our sample were observed just once and those with multiple observations have a relatively short ( $\leq 6$ months) baseline, which makes possible detection of only a small fraction of such binaries. Periods of $\mathrm{CH}$ stars, which are objects of similar nature (see below for details), can be as high as several years (see, e.g., Lucatello et al. 2005a).

To determine the expected number of mass-transfer binaries in a given population from first principles, one needs to know a number of properties of the underlying stellar population, such as the IMF, the binary fraction as well as binary period, eccentricity, and mass ratio distributions. Systematic studies of orbital properties of binary stars have been generally limited to the solar neighbourhood, and mostly deal with solar type (e.g. Duquennoy \& Mayor 1991) or M-dwarf stars (see, e.g., Fischer \& Marcy 1992; Abt \& Willmarth 2004). Nevertheless, reasonable assumptions can be made for these quantities. However, another critical ingredient is the range of periods (or semi-axes) within which mass transfer takes place effectively, which is highly uncertain, due to the current incomplete understanding of mass transfer during the common envelope phase and the treatment of angular momentum loss (for a discussion of the theory and examples of its applications, see Abate et al. 2013, 2015, respectively).

Alternatively, one can estimate the expected fraction of N-rich stars which result from transfer binaries in a more empirical way, by using the observed number of $\mathrm{CH}$ stars in the whole sample. Like Ba and CEMP-s stars, classical $\mathrm{CH}$ stars owe their peculiar composition to mass transfer from a relatively low-mass $(M \sim 1.5-$ $4 \mathrm{M}_{\odot}$ ) companion (see, e.g., McClure \& Woodsworth 1990; Lucatello et al. 2005a; Starkenburg et al. 2014). The mass range for the donor star is determined by the minimum mass for the third dredge-up (and hence for becoming a $\mathrm{CH}$ star) and by the onset of effective hot bottom burning, which burns $\mathrm{C}$ into $\mathrm{N}$ quite effectively.

Under the assumption that the binary incidence and the distributions of orbital period, mass ratio, and eccentricity are not dependent on the mass of the primary (which is quite reasonable in the mass range under discussion) and that the same mass-transfer physics applies, the ratio between the expected incidence of $\mathrm{CH}$ stars and $\mathrm{N}$-rich stars should be equal to the number ratio of donor stars in a given population. These numbers can be easily estimated by assuming that companions to $\mathrm{CH}$ stars and $\mathrm{N}$-rich stars had initial masses in the 1.5-3 and 3-8 $\mathrm{M}_{\odot}$ range, respectively. This ratio is $\sim 0.5$, being rather insensitive to whether one picks a Salpeter (1955), Kroupa (2001), or Chabrier (2003) IMF. This is likely an overestimate: while all stars in the $\sim 3-8 \mathrm{M}_{\odot}$ range undergo hot bottom burning and hence become enriched in nitrogen, the $\mathrm{Mg}-\mathrm{Al}$ cycle is activated at $T \sim 50 \mathrm{MK}$, and considerable Al production happens only for stars with masses within the higher end of the above range (with the lower cutoff depending on metallicity; see, e.g., Ventura et al. 2013). Using this ratio, we can determine, on the basis of the number of bona fide $\mathrm{CH}$ stars, the expected number of bona fide N-rich stars of binary origin.

In order to count the number of $\mathrm{CH}$-star candidates in our sample, a lower limit in $[\mathrm{C} / \mathrm{Fe}]$ must be defined. A commonly adopted number for metallicity within $-1 \lesssim[\mathrm{Fe} / \mathrm{H}] \lesssim 0$ is $[\mathrm{C} / \mathrm{Fe}]$ $=+0.3$ (see e.g. Lucatello et al. 2005b). However, [C/Fe] varies slightly with metallicity within the sample, so that a sample of $\mathrm{CH}$-star candidates defined on the basis of a constant lower limit would be biased towards stars with higher metallicity. Therefore, $\mathrm{CH}$-star candidates are selected in a manner analogous to how the $\mathrm{N}$-rich sample itself was defined, by fitting a high-order polynomial to the run of $[\mathrm{C} / \mathrm{Fe}]$ with $[\mathrm{Fe} / \mathrm{H}]$ and taking stars deviating from the fit by $+4 \sigma$. The $\mathrm{CH}$-star candidate sample defined in this way has a metallicity distribution in acceptable agreement with that of the N-rich stars (Section 3.6), which is consistent with the two samples belonging to the same underlying stellar population. The $\mathrm{CH}$-star candidate sample contains 52 stars. Examining the ASPCAP outputs for these stars, we find that 23 have one or more of several ASPCAP quality flags raised, namely STARWARN, ROTATIONWARN, CHI2 WARN, COLORTE WARN, and TEFF WARN. This indicates, as confirmed by visual inspection of several examples, that the spectral fits are of poor quality, or that the stellar parameters are unreliable, or both, suggesting that the abundances for these stars cannot be relied on (note that none of those flags were raised for any of the $\mathrm{N}$-rich stars). Eliminating these 23 stars from the sample and recalling that the expected ratio of $\mathrm{N}$-rich to $\mathrm{CH}$ stars resulting from binaries is $\sim 0.5$, our estimate of the number of N-rich stars owing their atmospheric composition to binary mass transfer would be $\sim 15$, or approximately a quarter of the sample identified in the previous sections.

We emphasize that this number is an upper limit, based on adopting a wide mass range for the production of $\mathrm{Al}$ in intermediatemass stars and a low cutoff for considering a star C-rich. In fact, we argue that the real number must be smaller, for the following reasons. First and foremost, one would expect to find a population of $\mathrm{N}$-rich stars in the Galactic disc if the phenomenon had an important contribution from mass-transfer binaries. There are $\sim 95$ $\mathrm{CH}$-star candidates, defined in the same way as above, in a disc sample defined by $|b|<20^{\circ}, 20^{\circ}<l<340^{\circ}$, and the same atmospheric parameters as the bulge sample. Of those, 46 have ASPCAP quality flags raised, leaving us with a sample of $49 \mathrm{CH}$-star candidates. Following the same reasoning as above, one would expect to find $\sim 25 \mathrm{~N}$-rich stars in the disc field, and instead not a single one is found. In fact, the few N-rich stars identified within the thusly defined disc belong to the low-latitude GCs M71 and NGC 6760. One might argue that, because the disc has a higher overall metallicity than the $\mathrm{N}$-rich sample, the production of $\mathrm{N}$ rich stars is inhibited, because models suggest that intermediatemass AGB stars produce less nitrogen at higher metallicity (Ventura et al. 2013). However, looking at the problem in a different way, we can examine the frequency of metal-poor N-rich stars in the bulge and ask how many such stars we would expect in the disc. At $[\mathrm{Fe} / \mathrm{H}]<-1.0$, there are $15 \mathrm{~N}$-rich stars out of a sample of 220 bulge stars in the same metallicity range and with same constraints on $[\mathrm{C} / \mathrm{Fe}]$. In the disc, the number of stars in the same locus of parameter space is 100 , so that one would expect about 7 $\pm 3 \mathrm{~N}$-rich moderately metal-poor disc stars to be discovered by APOGEE, in disagreement with the absence of any such stars in our sample. 


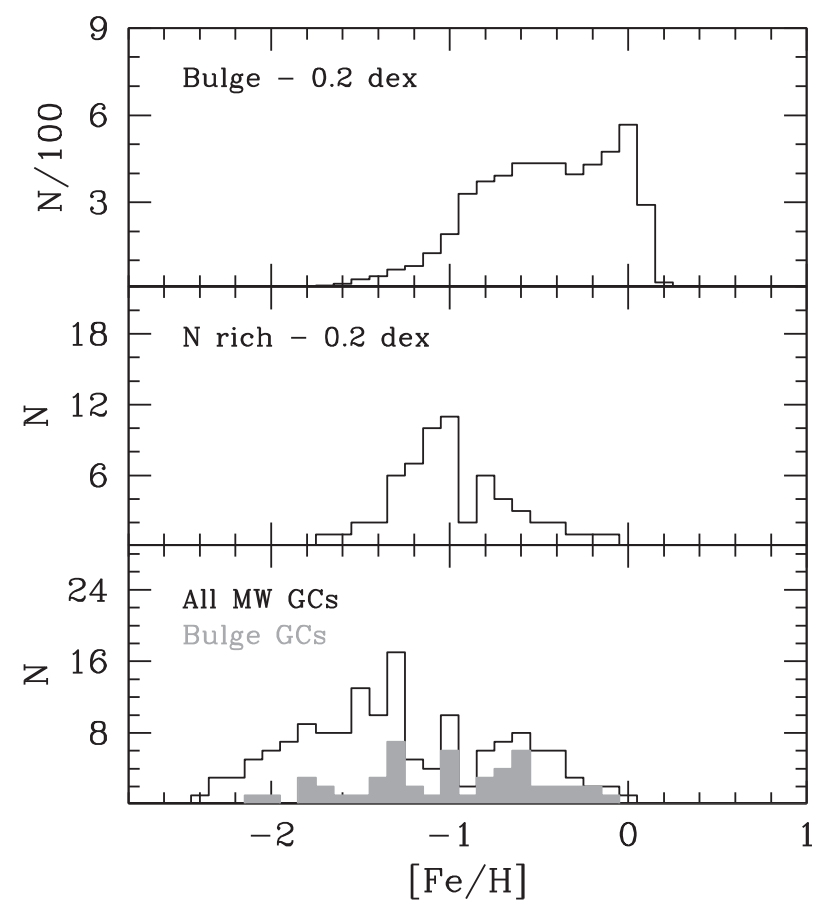

Figure 9. MDFs for the bulge field (top panel), N-rich stars (middle panel), and the Galactic GCs (bottom panel). The hatched grey histogram in the bottom panel shows the MDF obtained when only bulge GCs are considered. The MDFs of the bulge field and N-rich stars have significantly different shapes, so that it is difficult to conceive of a scenario where dissolved GCs contribute significant amounts of stellar mass to the Galactic bulge. The MDF of N-rich stars is also quite different from that of the Galactic GCs either considering the entire GC system (open histogram) or only those contained within the volume defined in Section 2 (hatched grey), making it difficult to conceive of a single mechanism connecting the population of dissolved GCs with the existing ones. See the text for details.

Therefore, we conclude that, to the best of our knowledge, the contamination of the N-rich star sample by the remnants of masstransfer binaries can amount to as much as 25 per cent, although the fraction is likely to be smaller.

We summarize the content of Sections 3.4 and 3.5 by concluding that there is no evidence for a high incidence of either AGB stars or mass-transfer binaries in our sample, so that the abundance pattern observed in most of the sample cannot be explained by those phenomena. We thus conclude that we have identified a stellar population in the inner Galaxy with a chemical composition akin to that of SG stars from GCs. The implications of this result are discussed in Section 4.

\subsection{Metallicity distribution}

In order to characterize the newly discovered stellar population, we examine its MDF and, in Section 4, contrast it with those of the Galactic bulge and GCs. Fig. 9 shows the MDFs for these three samples. The top panel shows the MDF of the entire population defined in Section 2, whereas the middle panel shows that of the $\mathrm{N}$-rich stars. The bottom panel shows the MDF of Galactic GCs included in the 2010 version of the Harris catalogue (Harris 1996). The metallicities of both APOGEE samples are corrected by -0.2 dex, to bring the APOGEE metallicity scale for $[\mathrm{Fe} / \mathrm{H}] \lesssim-0.4$ into agreement with the literature on abundance studies in the optical (see
Holtzman et al. 2015, for details). A constant correction is adopted for simplicity, even though it is only good for stars within the above metallicity range. As one can attest from inspection of fig. 6 of Holtzman et al. (2015), raw $[\mathrm{Fe} / \mathrm{H}]$ values provided by ASPCAP are in good agreement with the literature for metal-rich clusters, so that the corrected values for metal-rich are too low by 0.2 dex. Since the number of $\mathrm{N}$-rich stars in this metallicity regime is negligible, this small inaccuracy does not affect our results or conclusions.

\subsubsection{The bulge $M D F$}

A detailed examination of the MDF in the top panel of Fig. 9 is beyond the scope of this paper, and for that we refer the reader to García Pérez et al. (in preparation). For our purposes, we simply state that the global bulge MDF is in good agreement with those by Rojas-Arriagada et al. (2014) and Ness et al. (2013a), which are based on Gaia-ESO ${ }^{10}$ and ARGOS ${ }^{11}$ data, respectively. In particular, stars with $[\mathrm{Fe} / \mathrm{H}]<-1$ make up 5.8 and 4.6 per cent of the samples by Rojas-Arriagada et al. (2014) and Ness et al. (2013a), respectively, whereas they make up 228 out of our sample of 5140 stars, or $4.4 \pm 0.4$ per cent, which is in formal agreement with the Ness et al. (2013a) MDF.

To err on the side of caution, we checked for the presence of a bias against metal-poor giants that could have been introduced by the $T_{\text {eff }}<4500 \mathrm{~K}$ limit in our sample. We found that extending the sample by including giants as warm as $T_{\text {eff }}=6500 \mathrm{~K}$ added 18 stars to the sample, increasing only marginally the percentage of stars with $[\mathrm{Fe} / \mathrm{H}]<-1$, to $4.7 \pm 0.4$ per cent. Examining in detail the $T_{\text {eff }}$ distribution of the stars that are excluded by the $T_{\text {eff }}<4500 \mathrm{~K}$ cut, we find that all stars have $T_{\text {eff }}<5100 \mathrm{~K}$. The absence of warmer stars is due to the relatively shallow $(H=11)$ APOGEE magnitude limit in bulge fields, which restricts the sample to cool evolved giants. Regarding their metallicity distribution, the stars range between $-2<[\mathrm{Fe} / \mathrm{H}]<+0.0$, with a mean value of $[\mathrm{Fe} / \mathrm{H}]=$ -0.94 . On the metal-poor end, 16 stars have $[\mathrm{Fe} / \mathrm{H}]<-1$ to be contrasted with at sample of 220 stars in the same metallicity range and $3500<T_{\text {eff }}<4500 \mathrm{~K}$. Most importantly, only at most 5/18 of those stars can be classified as N-rich following the definition provided in Section 3.2. Of those, two have $[\mathrm{Fe} / \mathrm{H}]>-1$, two are within the $-1.5<[\mathrm{Fe} / \mathrm{H}]<-1$ interval, and one has $[\mathrm{Fe} / \mathrm{H}]<-1.5$. Such a small sample of warm N-rich stars has obviously no impact on the MDF of the N-rich population. It is thus safe to conclude that our sample is free of any important metallicity bias on the metalpoor end. In closing, we note that the $\mathrm{N}$-rich/N-normal ratio within this warm sub-sample is $5 / 18$, thus substantially higher than in the rest of the metal-poor sample. Given the considerable uncertainties in $[\mathrm{N} / \mathrm{Fe}]$ at such low metallicity and relatively high $T_{\text {eff }}$, we ascribe little significance to this result, while deeming it worthy of a closer examination in the near future.

\section{DISCUSSION}

The findings discussed in the previous section tantalizingly suggest that a population of stars with GC origin has been identified in the inner Galaxy. In line with early theoretical predictions (Tremaine, Ostriker \& Spitzer 1975, see also Gnedin et al. 2014), we hypothesize that these stars result from the destruction of pre-existing

\footnotetext{
${ }^{10}$ Gaia-ESO survey (Gilmore et al. 2012).

11 The Abundances and Radial velocities Galactic Origins Survey (Freeman et al. 2013).
} 
Galactic GCs. Interestingly, Brandt \& Kocsis (2015) proposed that millisecond pulsars, resulting from the destruction of GCs, can account for gamma-ray detections by the Fermi satellite towards the central regions of the Galaxy. This has important implications, as Brandt \& Kocsis (2015) show that their model does a better job of matching the data than models invoking annihilating dark matter (Hooper \& Goodenough 2011). Assuming that this hypothesis is correct, our discovery may also have interesting repercussions for the current understanding of the formation and evolution of the Galactic GC system, the presence of multiple stellar populations in GCs, and possibly also the formation of the bulge itself. In this section, we discuss some of these ramifications. We conduct our discussion in Sections 4.1 through 4.5 within the framework of a GC origin for the newly found stellar population. In Section 4.6, we speculate on a possible connection between N-rich stars and stellar populations inhabiting the cores of Andromeda and early-type galaxies. Finally, in Section 4.7, we discuss the origin of the new stellar population, conjecturing also possible scenarios beyond a pure GC origin.

\subsection{MDF constraints on the FG/SG ratio}

We begin by exploring the observed MDF in order to place constraints on the ratio between the numbers of stars with $\mathrm{N}$-normal and N-rich chemical compositions - the FG/SG ratio - in the parent population of N-rich stars. Fig. 9 shows that the MDF of N-rich stars (middle panel) differs quite substantially from that of the rest of the full sample (top panel). While the bulge MDF extends towards very high metallicities, that of $\mathrm{N}$-rich stars peaks around $[\mathrm{Fe} / \mathrm{H}]=-1$, with broad wings towards high and low metallicity. The apparent dip around $[\mathrm{Fe} / \mathrm{H}]=-0.9$ is strongly dependent on the binning adopted, so we assign no significance to it, given the small numbers per bin.

Assuming that the MDF of N-rich stars reflects precisely that of the destroyed GCs, one can use the metal-poor tail of the MDFs to constrain the ratio of $\mathrm{N}$-normal to $\mathrm{N}$-rich stars, and in this way derive an upper limit on the mass lost by GCs. There are $15 \mathrm{~N}$-rich stars with $[\mathrm{Fe} / \mathrm{H}]<-1$ out of a sample of 214 stars in the field within the same metallicity interval and $[\mathrm{C} / \mathrm{Fe}]<+0.15$. In other words, approximately 93 per cent of the stars in our sample with $[\mathrm{Fe} / \mathrm{H}]<-1$ have normal $\mathrm{N}$ abundances. Therefore, if one was to accept that all bulge stars with those metallicities originate from the destruction of GCs - a rather extreme scenario - one would conclude that 93 per cent of the stellar mass in those systems was originally in the form of FG stars. We emphasize that this is an upper limit. The MDF in the top panel of Fig. 9 shows that the inner Galaxy is dominated by a metal-rich stellar population which most likely does not result from GC destruction, seeing as it lacks a counterpart in the N-rich MDF. It is only reasonable to assume that some of those field populations not associated with GC destruction are also present at $[\mathrm{Fe} / \mathrm{H}]<-1$. Thus, to have all bulge stars with $[\mathrm{Fe} / \mathrm{H}]<-1$ come from GC destruction is most likely an unachievable feat. Consequently, the fraction of FG stars in those systems was most likely lower than 93 per cent. If we were to assume, for instance, that as much as $1 / 2$ of the mass in the volume sampled resulted from GC destruction, then we would conclude that FG/SG $\sim 6$ for the dissolved GCs. In subsequent sections, we explore the impact of the acceptable range of FG/SG ratio values on estimates of the total stellar mass in destroyed GCs. This result rules out models requiring FG/SG $\sim 10-100$ to address the mass budget problem.
Most importantly, this result poses a constraint on models that address the mass budget problem in GC formation by proposing that the FG/SG ratio was much larger in early GCs than observed today (e.g. D'Ercole et al. 2008; Schaerer \& Charbonnel 2011; Bekki 2011; Conroy 2012). Recently, Larsen, Strader \& Brodie (2012) and Larsen et al. (2014) determined integrated-light metallicities of GCs in dwarf galaxies, and established that the ratio between the stellar mass in GCs and the field, at the same metallicity, in the stellar haloes studied was too high to accommodate the requirements from selective stellar mass-loss models. That essentially the same conclusion has been reached from studies of samples in very different environments and metallicity ranges suggests that this may well be a general result.

\subsection{Mass in dissolved GCs}

Under the hypothesis that the N-rich stars result from dissolution of Galactic GCs, it is interesting to use their observed numbers to estimate the total stellar mass contained in dissolved GCs, to assess its contribution to the total stellar mass of the inner Galaxy and also to compare it with the mass contained in surviving GCs. However, only one type of GC star can be uniquely discriminated on the basis of the data under consideration - those belonging to the SG group. An estimate of the total stellar mass allocated in the form of these dissolved clusters obviously requires knowledge of the contribution by FG stars, which cannot be distinguished from field populations of the same metallicity, at least not on the basis of APOGEE data alone. In the absence of any constraints on the FG/SG ratio, we make assumptions for two limiting cases that hopefully bracket the entire range of possibilities. The minimal scenario assumes that there is no mass budget problem. In other words, the FG/SG ratio is exactly as observed today - about $1 / 2$ (Carretta et al. 2009). In contrast, in the maximal scenario, FG stars completely overwhelm N-rich stars. According to that scenario, early GCs were 10-100 times more massive in the past (Bastian \& Lardo 2015; Cabrera-Ziri et al. 2015), with essentially all the mass lost having been in the form of FGs. However, as discussed in Section 4.1, the FG/SG ratio cannot have been higher than $\sim 9 / 1$ without violating constraints from the low-metallicity end of the MDF discussed in the previous section.

We discuss these scenarios and their consequences in Sections 4.3 and 4.4.

\subsection{The minimal scenario}

To estimate the mass in dissolved GCs, we first estimate the fraction of the total stellar mass in the inner Galaxy contributed by N-rich stars, and following that we determine the contribution by dissolved GCs assuming FG/SG $=1 / 2$. The total number of N-rich stars, selected as described in Section 3.1, is 58 out of a total sample of 5140 stars. To first order, the ratio of N-rich to N-normal stars is not biased in any important way by APOGEE's target selection criteria (see Zasowski et al. 2013, for details), or by the definition of our sample, including its range of stellar parameters (Section 2). Therefore, we can safely state that N-rich stars amount to about 1.1 per cent of the total population in the inner Galaxy.

Assuming FG/SG $=1 / 2$, we conclude that the contribution of dissolved GCs to the stellar mass content of the inner Galaxy is small, at the 1.7 per cent level. By further assuming a (unlikely) scenario where the contribution of mass-transfer binaries to the $\mathrm{N}$ rich sample is at its maximum 25 per cent level, we would be led to conclude that the lower limit for the fractional contribution of dissolved GCs to the mass of the bulge is 1.3 per cent. 
The total mass of the Galactic bulge is estimated to be $\sim 2 \times 10^{10} \mathrm{M}_{\odot}$ (Sofue, Honma \& Omodaka 2009), with a stellar mass ranging somewhere between 1.25 and $1.6 \times 10^{10} \mathrm{M}_{\odot}$ (Portail et al. 2015). In the minimal scenario, the total mass in stars resulting from $\mathrm{GC}$ destruction would then range between 1.6 and $2.1 \times 10^{8} \mathrm{M}_{\odot}$, which is in relatively good agreement with model predictions for the contribution of disrupted GCs to the stellar mass contained within the inner few kpc of the Galaxy $\left(10^{7}-10^{8} \mathrm{M}_{\odot}\right.$, see, e.g., Tremaine et al. 1975; Gnedin, Ostriker \& Tremaine 2014). Brandt \& Kocsis (2015) showed that a similar mass in dissolved GCs can explain the gamma-ray detections by the Fermi satellite within $10^{\circ}$ of the Galactic Centre.

An alternative mass estimate can be obtained from consideration of a detailed model for the inner Galaxy, such as the one by Robin et al. (2014), which matches stellar counts in the 2MASS and SDSS catalogues. According to Robin et al. (2014), a cylinder of $2 \mathrm{kpc}$ radius and $4 \mathrm{kpc}$ height centred on the Galactic Centre contains a total $1.1 \times 10^{10} \mathrm{M}_{\odot}$. There are a total of 3244 APOGEE stars within the same volume, 45 of which belong to the N-rich population. Folding in factors accounting for the FG/SG ratio and maximum mass-transfer binary contribution, we conclude that the total mass of disrupted GCs would be $1.7 \times 10^{8} \mathrm{M}_{\odot}$, which is within the range of estimates provided above.

\subsubsection{Dissolved versus existing GCs}

Significantly, the mass contained in dissolved GCs is a factor of $\sim 6-8$ higher than the total mass in all existing Galactic GCs $\left(\sim 2.8 \times 10^{7} \mathrm{M}_{\odot} ;\right.$ Kruijssen \& Portegies Zwart 2009). This is obviously an important result. On the theory that N-rich stars are by-products of GC destruction, we would conclude that the Galactic GCs are remnants of a formerly much larger GC system - or of what would have become a much larger GC system today - that was largely destroyed through interaction with the environment.

This result naturally prompts us to ask whether such a dramatic destruction rate is a common phenomenon in the Universe, or whether the Milky Way is in some way special. Hudson, Harris \& Harris (2014) reported that the ratio $\eta$ between the mass of the GC system and total galaxy mass (including both dark and stellar matter) is $\sim 4 \times 10^{-5}$, and showed that it is constant over several orders of magnitude in galaxy mass and with a relatively small intrinsic scatter of only 0.2 dex. If the Galaxy underwent abnormally intense GC destruction, that should manifest itself by a substantial displacement from this mean value. Adopting the Galactic GC system mass from Kruijssen \& Portegies Zwart $\left(2009, \sim 2.8 \times 10^{7} \mathrm{M}_{\odot}\right)$ and a total mass of the Galaxy ranging between $6 \times 10^{11}$ and $3 \times 10^{12} \mathrm{M}_{\odot}$ (e.g. Barber et al. 2014; Fattahi et al. 2016), we obtain $\eta_{\text {MW }}$ ranging between $9 \times 10^{-6}$ and $5 \times 10^{-5}$. Considering the uncertainties in the numbers involved, one would conclude that $\eta_{\mathrm{MW}}$ is rather typical (if perhaps a bit on the low side), which suggests that GC destruction at the levels inferred from our results is a universal process. The fact that such a high rate of GC destruction is so finely tuned over a large range of galaxy masses and types is quite remarkable, and should be the subject of careful theoretical examination.

\subsubsection{Bulge, thick disc, or halo?}

As pointed out in Section 1, all components of the Galaxy contribute to the stellar mass within its inner few kpc. Thus, what we call our 'bulge' sample is in fact the superposition of all stellar populations lying within the range of Galactic coordinates and distances specified in Section 2 - which certainly includes halo, thin and thick disc, bar, and perhaps a classical bulge.

The MDFs in Fig. 9 provide clues as to the nature of the N-rich stars in our sample. As pointed out in Section 4.1, the MDF of the N-rich population and that of the rest of the sample are very different. The bulge MDF extends towards above solar metallicity, whereas that of the N-rich population peaks at $[\mathrm{Fe} / \mathrm{H}] \sim-1$, suggesting in fact an association with the thick disc or halo (e.g. Ness et al. 2013a; Robin et al. 2014). Examination of the incidence of $\mathrm{N}$-rich stars in other regions of the Galaxy can help decide between these two possibilities. An association with the Galactic halo or thick disc can be tested by searching for N-rich stars in other parts of these Galactic components, and checking whether the observed numbers match expectations based on the frequency of $\mathrm{N}$-rich stars in the inner Galaxy. We start by comparing our numbers with those obtained by other groups from analysis of SDSS-SEGUE data for halo stars at larger Galactocentric distances. Martell et al. (2011) and Schaerer \& Charbonnel (2011) estimated the total contribution of GC stars to the halo mass budget (10-20 per cent). Recall that our estimate for the mass in the dissolved GCs in the inner Galaxy is $\sim 1.6-2.1 \times 10^{8} \mathrm{M}_{\odot}$, according to the minimal scenario (Section 4.3). According to the Besançon models (Robin et al. 2012, 2014), the Galactic halo accounts for $\sim 8.5 \times 10^{8} \mathrm{M}_{\odot}$ within the inner $2 \mathrm{kpc}$ of the Galaxy (Section 4.3.2), so that in the minimal scenario dissolved GCs contribute $\sim 19-25$ per cent of the stellar halo mass, which is very similar to the estimates by Martell et al. (2011) and Schaerer \& Charbonnel (2011). However, those authors assumed a much larger primordial FG/SG ratio, which is far more compatible with that adopted for our maximal scenario (see below). Assuming that there is no strong reason for one to adopt different FG/SG ratios for inner and outer halo, one would end up with a substantial variation in the contribution to the halo stellar mass by GC stars as a function of Galactocentric distance. Adopting the FG/SG ratio from the minimal scenario, the contribution to the halo mass inferred from Martell et al. (2011) and Schaerer \& Charbonnel (2011) would be reduced by a factor of a few to several. Indeed, in a more recent effort based on APOGEE DR12 data, Martell et al. (2016) searched for N-rich stars in high-latitude halo fields adopting a definition that is consistent with that described in Section 2. From a resulting sample of five halo N-rich stars, they concluded that, adopting an FG/SG ratio consistent with our minimal scenario, the contribution of dissolved GCs to the halo mass would be $\sim 4$ per cent. In conclusion, there are fewer N-rich stars in the DR12 APOGEE halo sample, by a factor of $\sim 5$, than expected if the frequency of those stars was the same across the entire halo.

The above approach suffers from a basic limitation stemming from the small relative size of the APOGEE halo sample. We exploit the much larger APOGEE sample at low Galactic latitudes for a statistically more robust comparison between expected and observed $\mathrm{N}$-rich star numbers. For that purpose, we perform the following exercise. We first use models to estimate the 'component-specific' frequency of $\mathrm{N}$-rich stars under the assumption of their association with each of those components, then use that frequency to estimate the expected number of N-rich stars in other regions of the Galaxy. By 'component-specific' frequency, we mean the fraction of the halo or thick-disc stars that are N-rich if the N-rich stars found in the inner Galaxy are assumed to be associated entirely with either of those components. This estimate requires knowledge of the breakdown of the stellar mass in the inner Galaxy among various Galactic components. For that purpose, we adopt the Besançon models by 
Table 1. Expected numbers of $\mathrm{N}$-rich stars with $[\mathrm{Fe} / \mathrm{H}]<-0.7$ in other fields.

\begin{tabular}{lcccc}
\hline & $f$ & $f_{\mathrm{Nr}}$ & $f_{\text {bulge }}$ & $N_{\text {exp }}$ \\
\hline Halo & 0.021 & $0.07 \pm 0.01$ & 0.18 & $6 \pm 1.5$ \\
Thick disc & 0.920 & $0.38 \pm 0.08$ & 0.85 & $29 \pm 7$ \\
\hline
\end{tabular}

Robin et al. (2012, 2014), which match stellar counts in 2MASS and SDSS by considering a combination of four components: a thick disc, a thin disc, a halo, and a bar. No classical bulge was needed in order to fit the data for the inner Galaxy. The mass breakdown among the various components within a cylinder with $2 \mathrm{kpc}$ radius and $4 \mathrm{kpc}$ height located at the Galactic Centre is as follows:

(i) thick disc: $5.7 \times 10^{9} \mathrm{M}_{\odot}$;

(ii) bar: $4.3 \times 10^{9} \mathrm{M}_{\odot}$;

(iii) halo: $8.5 \times 10^{8} \mathrm{M}_{\odot}$;

(iv) thin disc: $1.1 \times 10^{8} \mathrm{M}_{\odot}$

Next, using survey simulations based on the same models, we calculate how many N-rich stars are expected in the APOGEE sample of low-latitude fields with $|b|<20^{\circ}$ and $20^{\circ}<l<340^{\circ}$ if they were associated with either thick disc or halo. The expected number of N-rich stars integrated within all that area of sky is given by

$N_{\text {exp }}=N_{\text {obs }} F(\mathrm{H} \mid \mathrm{TD}) f_{\mathrm{Nr}}(\mathrm{H} \mid \mathrm{TD})$

where $N_{\text {obs }}$ is the total number of stars observed by APOGEE within that area and following the selection criteria described in Section 2, $F(\mathrm{H} \mid \mathrm{TD})$ is the fraction of those stars belonging to either the thick disc or the halo, and $f_{\mathrm{Nr}}(\mathrm{H} \mid \mathrm{TD})$ is the component-specific frequency of $\mathrm{N}$-rich stars in that area of the sky. We take $F(\mathrm{H} \mid \mathrm{TD})$ from survey simulations based on the Besançon models and estimate $f_{\mathrm{Nr}}(\mathrm{H} \mid \mathrm{TD})$ from a combination of the observed frequency of $\mathrm{N}$-rich stars in our bulge fields and model estimates for the contribution of thick-disc and halo stars to the volume sampled, so that

$f_{\mathrm{Nr}}(\mathrm{H} \mid \mathrm{TD})=\frac{N_{\text {bulge }, \mathrm{Nr}}}{N_{\text {bulge }} f_{\text {bulge }}(\mathrm{H} \mid \mathrm{TD})}$,

where $N_{\text {bulge }}$ is the number of stars observed within the APOGEE bulge fields, $N_{\text {bulge, } \mathrm{Nr}}$ is the number of those among the latter who are $\mathrm{N}$-rich, and $f_{\text {bulge }}(\mathrm{H} \mid \mathrm{TD})$ is the component-specific frequency of $\mathrm{N}$ rich stars in the inner Galaxy, according to survey simulations. For this exercise, we limit the sample to stars with $[\mathrm{Fe} / \mathrm{H}]<-0.7$, where the contribution by halo and thick-disc populations is maximal.

In Table 1, numbers for the quantities defined in equations (1) and (2) are provided. The total number of stars in the APOGEE sample within $20^{\circ}<l<340^{\circ}$ and $|b|<20^{\circ}$ with stellar parameters as defined in Section $2^{12}$ and $[\mathrm{Fe} / \mathrm{H}]<-0.7$ is $N_{\text {obs }}=476$. The size of the APOGEE bulge sample in the same metallicity range is $N_{\text {bulge }}=742$, and the number of N-rich stars is $N_{\text {bulge, } \mathrm{Nr}}=42$.

The numbers in Table 1 inform us that, if our N-rich stars were associated with the thick disc, roughly $30 \mathrm{~N}$-rich stars should have been detected in other Galactic longitudes. The number in the case of an association with the halo is much smaller, of the order of 6. As mentioned elsewhere, no N-rich stars were found anywhere in the Galactic regions considered in this exercise. These numbers tempt the assertion that a halo association is more likely, as the total number of detections predicted in that case is substantially

\footnotetext{
${ }^{12}$ The simulations were actually carried out assuming $\log g=2$, but that has no impact on our results, given APOGEE's relatively bright $(H=11.2)$ magnitude limit in bulge fields.
}

smaller, and thus closer to the observed number (zero); however, both estimates are off from the observed values by $4 \sigma$. Such a substantial discrepancy may be simply due to our assumption that $f_{\mathrm{Nr}}$ inferred in the inner Galaxy applies everywhere else. Previous work has indeed suggested the presence of a possible gradient in the incidence of N-rich stars (Carollo et al. 2013) in the Galactic halo. Considering also the fact that stars with SG abundances have been found in the halo (e.g. Carretta et al. 2010; Martell et al. 2011; Carollo et al. 2013; Lind et al. 2015; Fernandez-Trincado et al. 2016), it seems natural to conclude that an association of our N-rich population with the inner halo seems more likely than with the thick disc. The discrepancy with the numbers expected in other regions of the halo (e.g. Martell et al. 2011, 2016) indicates that the frequency of N-rich stars may be higher in the inner halo, which is in and of itself an important constraint on models for the origin of this population.

Finally, a by-product of the above exercise is an assessment of the contribution of dissolved GCs to the stellar mass budget of those Galactic components in the case where the N-rich stellar population is associated with either of them. We have seen in Section 4.3 that, according to the minimal scenario and assuming maximum contribution to the N-rich sample by binary stars, dissolved GCs contribute $1.6-2.1 \times 10^{8} \mathrm{M}_{\odot}$ to the volume sampled by the above model. Assuming an association with the Galactic halo, we would conclude that dissolved clusters contribute $\sim 19-25$ per cent to the stellar mass of the halo within about $2 \mathrm{kpc}$ of the Galactic Centre. Conversely, if $\mathrm{N}$-rich stars are members of the thick disc, their contribution to the total mass of that Galactic component would range between 2.8 and 3.7 per cent.

\subsection{The maximal scenario}

We now consider the maximal scenario, according to which GCs were much more massive in the past and the vast majority of the mass lost was in the form of FG stars. In that scenario, SG stars, such as the N-rich population reported in this paper, are but a trace of the total GC population. According to some models, to satisfy observations of stellar abundances in Galactic GC members, GCs would have to have been 10-100 times more massive in the past (for references, see, e.g., Gratton et al. 2012; Bastian \& Lardo 2015; Cabrera-Ziri et al. 2015), which would lead us to conclude that the bulk, if not all, of the stellar mass of the bulge or inner Galaxy resulted from the dissolution of GCs similar to the ones that populate the Galactic halo, bulge, and thick disc today, or their parent systems. According to that scenario (Schaerer \& Charbonnel 2011; Gratton et al. 2012), the presence of SG stars in the field of the halo (Martell \& Grebel 2010) would similarly imply that a substantial fraction of the halo was also built from dissolution of GCs (Carollo et al. 2013).

To begin with, the MDFs of N-rich stars and the rest of the inner Galaxy population are difficult to reconcile with the premise that dissolved GCs contribute importantly to the total stellar mass in the spatial region sampled in this work. One possible way to salvage that proposition is by assuming that the FG/SG in GCs increases substantially towards high metallicity. Statistics on $\mathrm{C}-\mathrm{N}$ anticorrelations in metal-rich $([\mathrm{Fe} / \mathrm{H}] \gtrsim-0.5)$ GCs are currently meagre, which makes it difficult to test the hypothesis. However, Carretta et al. (2010) reported that there is tentative evidence for the presence of a correlation between GC metallicity and the extension of the Na-O anticorrelation and, by association, the incidence of N-rich-like, SG stars in Galactic GCs. Moreover, studying M31 GCs in integrated light, Schiavon et al. (2013) established a 
correlation between mean $[\mathrm{N} / \mathrm{Fe}]$ and $[\mathrm{Fe} / \mathrm{H}]$, again arguing for an enhancement, rather than a diminution, in the relative number of N-rich stars in more metal-rich GCs. Therefore, if GCs had contributed substantially to the mass of the bulge, we would expect the N-rich population to have a much more metal-rich MDF. Therefore, we conclude that, at face value, the MDFs suggest that it is unlikely that dissolved GCs contribute significantly to the total stellar mass within the volume sampled in this study.

An important contribution of dissolved GCs to the stellar mass budget is also likely incompatible with current understanding of the structure and stellar content of the inner Galaxy. Regarding stellar population content, there may be small, but non-negligible, differences in abundance pattern between FG stars in existing GCs and both halo and bulge field populations. For instance, fig. 3 of Carretta et al. (2009) hints at the possible existence of small [O/Fe] differences between field and GC populations in the halo, and larger ones in the bulge. Whether these are real or due to systematics stemming from differences in data quality and/or analysis methods is not clear. As regards the structure of the bulge, a substantial part of its stellar mass is seemingly contained in the bar (e.g. Ness et al. 2013a; Rojas-Arriagada et al. 2014). Indeed, based on the Besançon models (Robin et al. 2014), one would estimate that the bar contributes $\sim 40$ per cent of all stellar mass within $2 \mathrm{kpc}$ of the Galactic Centre, which implies that a considerable part of the bulge population probably resulted from secular evolution of the disc.

The above caveats aside, in this section we define the maximal scenario in such a way that the constraints from low-metallicity stars discussed in Section 4.1 are met. The resulting numbers are then used to compare the total mass in dissolved GCs with that of the existing Galactic GCs, and also to estimate the maximal contribution of dissolved GCs to the stellar mass budget of various Galactic components. The key constraint posed by the MDF of $\mathrm{N}$-rich stars of $[\mathrm{Fe} / \mathrm{H}] \lesssim-1$ is that the $\mathrm{FG} / \mathrm{SG}$ ratio in dissolved GCs cannot exceed 0.93 . Thus, if the ratio between $\mathrm{N}$-normal and $\mathrm{N}$-rich stars in dissolved GCs is maximum, then the $45 \mathrm{~N}$-rich stars contained in the cylindrical volume considered in Section 4.3 correspond to only 7 per cent of the mass in that volume. Therefore, the total number of stars resulting from GC destruction in our sample should be 843 . Considering that our sample contains 5140 stars, the maximal scenario implies that $\sim 16$ per cent of all stars in our bulge sample result from dissolution of Galactic GCs. In this scenario, the mass in dissolved GCs outnumbers that of the existing systems by a factor of $\sim 60-90$, depending on the total mass adopted for the inner Galaxy (Section 4.3). If the numbers used considered only stars within the cylindrical volume included in the Besançon model calculations, $\sim 20$ per cent of the stars in that cylinder would have resulted from $\mathrm{GC}$ dissolution, leading to a total mass that outweighs the GC system by a factor of 80 .

To estimate the contribution of dissolved GCs to the stellar mass in the halo or thick disc, we simply compare the latter percentage with those based on the Besançon models, listed in Section 4.3.2. According to those models, the Galactic halo accounts for $\sim 8$ per cent of the stellar mass in the inner Galaxy, so that the maximal mass in dissolved GCs outweighs that in the inner halo by over a factor of 2. Conversely, considering an association of $\mathrm{N}$-rich stars with the thick disc, which according to the Besançon models accounts for $\sim 50$ per cent of the mass in the inner Galaxy, one would be led to conclude that the $\sim 40$ per cent of the mass in the thick disc results from GC dissolution. According to the maximal scenario, the halo alone cannot contribute to the whole mass of dis- solved GCs, with the thick disc contributing an important fraction, if not all of it.

In conclusion, the evidence accumulated thus far seems to suggest that the maximal scenario is ruled out by the data, and the FG/SG ratio in GCs was much lower than the 93 per cent limit described above. The latter implies that the contribution of dissolved GCs to the halo mass inferred in previous studies is overestimated. It seems safe to conclude that the fraction of halo stellar mass contributed by dissolved GCs peaks towards the inner halo, but the exact number is hard to pin down due to uncertainties in the FG/SG ratio, in the contribution of the inner halo to the stellar mass within the inner few kpc of the Galaxy, and in the association of the N-rich stars with any of the overlapping components of the Galaxy in its inner regions.

\subsection{MDFs of N-rich stars versus Galactic GCs}

We now examine the comparison between the MDFs of the N-rich stars and the Galactic GCs, shown in the bottom panel of Fig. 9. As is well known, the Galactic GC MDF is seemingly bimodal, showing evidence for the presence of two peaks, at $[\mathrm{Fe} / \mathrm{H}] \sim-1.6$ and -0.7 , with a trough at $[\mathrm{Fe} / \mathrm{H}] \sim-1$, which is precisely where the peak of the N-rich MDF is located. Low-number statistics prevents an analysis of the shape of the N-rich star MDF. For instance, as noted above, the apparent trough in the MDF of the N-rich population at $[\mathrm{Fe} / \mathrm{H}] \sim-0.9$ has no statistical significance. Nevertheless, despite the relatively low numbers, much can be learned from comparison between the N-rich star MDF and that of the GC system. The range encompassed by the $\mathrm{N}$-rich $\mathrm{MDF}$ goes from $[\mathrm{Fe} / \mathrm{H}] \sim-1.5$ to almost solar. In comparison, the GC MDF extends towards metallicities as low as $[\mathrm{Fe} / \mathrm{H}] \sim-2.5$. It is unclear whether the lack of stars with $[\mathrm{Fe} / \mathrm{H}] \lesssim-1.5$ in the $\mathrm{N}$-rich $\mathrm{MDF}$ is real or due to low-number statistics. As discussed in Section 3.6.1, the APOGEE sample studied in this paper presents no important bias against metal-poor stars.

The Galactic GC MDF differs substantially from that of the $\mathrm{N}$-rich population even when only GCs within the volume containing the N-rich sample (Section 2) are considered, as indicated by the hatched grey histogram in the bottom panel of Fig. 9. Despite the relatively low numbers, the bulge GC MDF is markedly different from that of the N-rich population, with no peak at any particular metallicity. A Kolmogorov-Smirnov test strongly rejects the hypothesis that the two samples are drawn from the same parent population, at a level $P=0.002$.

It is clear from the above that the N-rich star MDF does not match that of the existing GC system, either considered as a whole or taking the halo and bulge/thick-disc components separately. We interpret this result as evidence that evaporation of existing Galactic GCs has not contributed significantly to the population of N-rich stars. The rationale behind this interpretation is that it would be difficult, without tidal evaporation that is strongly dependent on metallicity, to deplete the masses of an original population of GCs by an order of magnitude, while completely obliterating its MDF. Tidal evaporation is of course likely to have operated differently on GCs of different metallicity, because more metal-rich GCs, typically located at smaller Galactocentric distances, were probably subject to more vigorous tidal evaporation. In that case, we would possibly expect the N-rich MDF to resemble more strongly that of thick disc/bulge GCs, which we show not to be the case. In short, the MDFs require destruction to have been very efficient for GCs in a narrow metallicity range around $[\mathrm{Fe} / \mathrm{H}] \sim-1$, and less efficient everywhere else, which seems contrived. 
One additional possibility is that the MDF of the Galactic GC system is not itself bimodal, but rather suffers from non-linearity effects such as those claimed by Yoon, Yi \& Lee (2006) to affect the conversion between integrated colours and metallicity of extragalactic GC systems. While giving it a nod in this paper, we choose to defer this line of reasoning to a future study, and take the Galactic GC MDF from Fig. 9 at face value. We therefore suggest that the $\mathrm{N}$-rich population, if at all associated with GCs, was predominantly produced by the destruction of a large population of early Galactic GCs. In fact, if indeed the halo and bulge/disc components of the Galactic GC system had different origins (e.g. Shapiro, Genzel \& Förster Schreiber 2010; Tonini 2013), it is also likely that their destruction efficiencies were different, so that one would indeed expect that the MDFs of the destroyed and surviving populations differ. In Section 4.7, we discuss possible scenarios for the origin and fate of this population of GCs, the remnants of which we seem to have discovered in the inner Galaxy.

\subsection{Dissolved GCs in the cores of other massive galaxies?}

While GCs and their parent populations do not seem to be important building blocks of the Galactic bulge, the situation may be different in other environments. Unlike the inner Galaxy, where the bar and thick disc contribute $\sim 91$ per cent of all stellar mass (Section 4.3.2), the cores of early-type galaxies are dominated by a spheroidal component, which like the Galactic halo is the result of accretion of low-mass stellar systems. We have seen from the discussion above that the evidence points to the $\mathrm{N}$-rich population being associated with the Galactic halo, in which case dissolved GCs contribute a minimum 19-25 per cent of the stellar halo mass. If dissolved GCs contribute a similar fraction to the stellar mass in the cores of early-type galaxies, one would expect the mean abundances inferred from integrated-light studies to be influenced by the chemical compositions typical of N-rich stars. In that case, the abundances of elements such as nitrogen, sodium, and aluminium could be enhanced in the integrated spectra of early-type galaxies.

Interestingly, integrated-light studies have shown that early-type galaxies are characterized by large mean values of $[\mathrm{N} / \mathrm{Fe}]$ and $[\mathrm{Na} / \mathrm{Fe}]$, which are moreover strongly correlated with galaxy mass and velocity dispersion (Graves et al. 2007; Schiavon 2007; Conroy, Graves \& van Dokkum 2014; Worthey, Tang \& Serven 2014; Smith et al. 2015). Moreover, in a recent study, Zieleniewski et al. (2015) estimated $[\mathrm{Na} / \mathrm{Fe}] \gtrsim+0.3$ within the inner $\sim 0.4 \mathrm{kpc}$ of the Andromeda galaxy, with $[\mathrm{Na} / \mathrm{Fe}]$ possibly increasing to as much as +1.0 within the inner $\sim 40 \mathrm{pc}$. Although difficult to disentangle from IMF effects on the Na I $\lambda 8200 \AA$ line (e.g. Spinrad \& Taylor 1971; Schiavon, Barbuy \& Bruzual 2000; van Dokkum \& Conroy 2010), this is a strong indication of the presence of $\mathrm{Na}$ enhanced populations in the core of Andromeda. Along the same lines, Burstein et al. (1984) showed (zero-point uncertainties aside; see Schiavon et al. 2012a) that CN bands in the core of M31 are enhanced, being consistent with their strengths in the integrated spectra of M31 and Galactic GCs. The work by Strader et al. (2013) is also worthy of notice in this context. They analysed the optical spectrum of an ultracompact dwarf galaxy satellite of M60, in the Virgo cluster, and found very high $[\mathrm{N} / \mathrm{Fe}]$ and $[\mathrm{Na} / \mathrm{Fe}]$ abundance ratios.

Along a different line of evidence, it has been known for decades that the spectral energy distributions of early-type galaxies are characterized by a strong ultraviolet (UV) excess (Code \& Welch 1979; Brown et al. 1997; Greggio \& Renzini 1999; O’Connell 1999; Yi et al. 2005). There is general agreement that extreme horizontal branch stars (EHBs) are the main source of UV radiation in those systems (e.g. O'Connell 1999). The EHBs have been identified in resolved UV images of the cores of M31 and M32 (Brown et al. 1998, 2000) and are also found in large numbers in Galactic GCs (e.g. Dorman, O'Connell \& Rood 1995; Schiavon et al. 2012b). If GCs make an important contribution to the stellar mass in the cores of early-type galaxies, one would expect them to contribute significantly to their EHB content as well (see Bekki 2012, for a model along those lines).

We speculate that these results indicate the presence of a population of dissolved GCs in the core of Andromeda, and possibly also in the central regions of early-type galaxies. The population of $\mathrm{N}$-rich stars we discovered in the heart of the Galaxy may thus be the tracers of a global phenomenon associated with the formation of spheroidal systems in general.

\subsection{Origin of the presumptive population of dissolved GCs}

The question of the origin of N-rich stars is inevitably tangled with two major unsolved problems in galaxy formation, namely formation of the Galactic bulge (and galaxy bulges in general) and GC formation. In this sub-section, we briefly discuss four possible scenarios to explain the origin of N-rich stars. The first two scenarios assume, as usual, that N-rich stars were initially formed within, and later lost to, the gravitational potential of GCs. A third scenario relaxes this assumption and contends that instead N-rich stars were formed within the same molecular clouds as GCs, but were never gravitationally bound to them. A fourth scenario suggests that N-rich stars were instead never necessarily associated with GCs and are rather the oldest existing stellar population formed in the Galactic bulge itself.

\subsubsection{GC origin}

It is now generally agreed that most GCs formed in giant molecular clouds (GMCs) generated by disc instabilities in galaxies at $z \gtrsim 2$ (e.g. Kravtsov \& Gnedin 2005; Shapiro et al. 2010; Tonini 2013; Kruijssen 2014, 2015; Rossi \& Hurley 2015). GC formation motivated by mergers of gas-rich galaxies is another possible mechanism (Ashman \& Zepf 1992; Muratov \& Gnedin 2010), but one thought to make a minor contribution to the total stellar mass allocated in GC systems today (Kravtsov \& Gnedin 2005).

Elmegreen \& Hunter (2010) championed the notion that newborn clusters are efficiently destroyed by tidal interaction with GMCs, which are present in the very environment that initially gave birth to clusters - the so-called 'cruel cradle effect' (see also Kruijssen et al. 2011, 2012). Kruijssen $(2014,2015)$ proposed a model, based on an analytical formulation, where GC formation takes place in two phases. In the first phase, GCs are formed with a power-law mass distribution, within GMCs hosted by turbulent discs at $z \sim 2-$ 3. Formation is followed quickly by vigorous disruption due to tidal interaction with GMCs. Since tidal destruction of lower mass clusters is more efficient, the original power-law mass function is converted into the lognormal distribution observed today. Survival of GCs is ultimately dependent on the occurrence of a galaxy merger, which removes them from their inhospitable birthplaces. Mergers trigger a second phase, during which GCs are incorporated into the halo of a new host galaxy or merger remnant, where they suffer a more gentle, longer tidal evaporation through interaction with the gravitational potential of the new host galaxy. This scenario matches a number of properties of GC systems, including the GCspecific frequency and mass distribution in galaxies with a range of 
metallicities and halo/stellar masses. It is also in line with previous suggestions that the disc/bulge GCs were formed in situ (Shapiro et al. 2010), whereas the halo GC system has largely been accreted (Tonini 2013).

This scenario for GC formation suggests that there may be at least two possible channels for the production of $\mathrm{N}$-rich stars involving GC destruction: (i) the in situ $G C$ channel, whereby these stars originate from the population of GCs that migrated into the inner Galaxy from an early turbulent Galactic disc, being destroyed in the process; (ii) the ex situ $G C$ channel, according to which these stars originate from the dissolution of a population of accreted GCs. We briefly discuss these formation channels below.

(i) In situ GC origin. Bournaud, Elmegreen \& Elmegreen (2007) and Elmegreen, Bournaud \& Elmegreen (2008) proposed that the Galactic bulge was formed by the coalescence of giant clumps hosted by turbulent discs at high redshift (see review by Bournaud 2016). Those clumps were also the sites of GC formation, as proposed by various authors cited above. According to this scenario, one would expect that GCs initially formed in the disc and eventually migrated towards the inner Galaxy, with some of them losing mass and/or being destroyed in the process, and others surviving in the Galactic thick disc and bulge. In that scenario, the GCs associated with the thick disc and bulge of the Galaxy today would be remnants of an active past of star formation in the Galactic disc (Shapiro et al. 2010). Presumably, this process would naturally result in the presence of the by-products of GC dissolution in the inner Galaxy, as reported in this paper. Moreover, one would also expect such populations to be found in the Galactic disc, as a considerable amount of tidal destruction is expected to have taken place during interaction between GCs and GMCs in the disc (Kruijssen 2015).

As reported in Section 4.3.2, a search for N-rich disc stars in the APOGEE DR12 data base, adopting the same parameters as described in Section 2, but focusing instead on $20^{\circ}<l<340^{\circ}$, resulted in no field stars with an $\mathrm{N}$-rich abundance pattern. This result does not necessarily mean that $\mathrm{N}$-rich stars do not exist in the disc, since, despite its very large sample, the APOGEE coverage of the disc is of course limited. Moreover, one would in any case expect relative numbers in the disc to be lower than in the inner Galaxy, given that the disc has been subject to a much longer history of star formation at later times, for several Gyr, where conditions did not favour formation of GCs, or their parent systems (Kruijssen 2015), leading to a decrease of the ratio of $\mathrm{N}$-rich/N-normal stars.

As discussed in Section 3.6, the MDFs of the N-rich stars and thick disc/bulge GCs are quite different, with the former peaking at $[\mathrm{Fe} / \mathrm{H}] \sim-1$ and the latter spanning a wide range of metallicities and not peaking at any particular value. Shapiro et al. (2010) pointed out that the metallicities of star-forming clumps in $z \sim 2-3$ discs were high and thus not compatible with the formation of the metalpoor component of the Galactic GC system. It is unclear whether current models for formation of GCs in turbulent discs can account for the existence of the metal-poor GCs and N-rich stars seen in the inner Galaxy. Shapiro et al. (2010) suggest that metal-poor GCs may have formed along cold filaments that were proposed by Dekel et al. (2009) and others to account for high star formation rates in the early Universe. Whether the MDF of the existing GCs and that of those that were dissolved in the past can be accounted for in detail by these models is an open question. Another possibility for the formation of these metal-poor GCs is discussed next.

(ii) Ex situ GC origin. Following Kruijssen (2015), GCs can also have been formed in lower mass galaxies, in much the same way as described above, and later accreted with their host galaxy into the deeper potential well of the Galactic dark matter halo, where they could have been subject to tidal evaporation or destruction. There is abundant evidence for the formation of the Galactic halo itself, and that of the nearest giant spiral galaxy, Andromeda, through accretion of satellite galaxies (e.g. Searle \& Zinn 1978; Ibata, Gilmore \& Irwin 1994; Belokurov et al. 2006b; Law \& Majewski 2010; Gilbert et al. 2014; Ibata et al. 2014). Moreover, the tidal evaporation of halo GCs through interaction with the Galactic potential has been spotted in real time (e.g. Rockosi et al. 2002; Odenkirchen et al. 2003; Belokurov et al. 2006a), and must be responsible for at least part of the population of N-rich stars detected in the halo by Martell \& Grebel (2010) and Martell et al. (2011), and possibly also for a small fraction of those found in the inner Galaxy. However, tidal evaporation of this nature is probably too inefficient (Baumgardt \& Makino 2003; Kruijssen 2015) to account for a substantial fraction of the N-rich stars detected in the inner Galaxy. Indeed, it is thought that GCs accreted in this process make up the blue/metalpoor component of GC bimodal distributions in galaxies (Tonini 2013), so that their characteristic metallicities are typically lower than those of the N-rich stars identified in the inner Galaxy in this study (cf. Fig. 9). However, if one accepts the scenario whereby GCs are formed in clumpy discs at $z \gtrsim 2$, then it is possible that low-mass galaxies accreted to the early Galaxy hosted a population of GCs that were previously dissolved/stripped in their parent turbulent discs, and were later incorporated into the field of the Milky Way itself. Dynamical friction could then drive the stars - including those of the N-rich variety - belonging to the most massive of those systems, which potentially host the most metal-rich GCs, towards the central parts of the Galaxy, where they reside today.

\subsubsection{Shared nursery origin}

The present paper reports the finding that the vast majority of the stars with a so-called SG star abundance pattern appear to reside in the field, not in clusters (Section 4.2). This result quite naturally invites one to question the standard assumption that these stars were formed in GCs to begin with. Indeed, the fact that they were first discovered in GCs is due to a severe observational bias and does not imply a sine qua non genetic link - at least not until detailed physical models of GC formation account for their existence in a quantitative sense, by matching the extant data sets. The stark disagreement between the MDF of the N-rich stars and that of the Galactic GC system - modulo possible zero-point differences lends further support to the notion that the two populations do not share the same origin.

In light of this evidence, a model that can form N-rich stars outside the gravitational well of GCs may be required. The natural sites for the formation of such field N-rich stars would be the very molecular clouds that formed the GCs. It is conceivable that not all stars formed in those clouds ended up in GCs - indeed, the evidence from studies of molecular clouds in the Galaxy point in the opposite direction (e.g. Longmore et al. 2014). Stars not bound to GCs would be lost to the field quite easily, thus making up the majority of the field population observed today. One could imagine a scenario where the ratio between GC bound and unbound stars formed in such molecular clouds is a function of the physical conditions in the cloud (e.g. density, chemical composition) as well as the environment. Such a scenario could potentially explain the predominance of $\mathrm{N}$-rich stars that are currently not gravitationally bound to any Galactic GC, without the need to invoke efficient 
GC destruction. Open clusters, having been formed under physical conditions that are rather different from those that gestated GCs, were not able to produce stars with an SG abundance pattern. If molecular clouds existed that formed N-rich stars, but no GCs, this scenario would naturally account for the mismatch between the MDFs of the N-rich stars and the existing Galactic GCs. Moreover, if this strawman scenario is correct, one would expect to find $\mathrm{N}$-rich stars in the same environments as GCs today, seeing as at least some of them would have been formed in the same molecular clouds as the GCs themselves. As discussed above, N-rich stars were indeed discovered in the halo (Martell et al. 2011; Carollo et al. 2013) and in the inner Galaxy (this study), but it is noteworthy that none so far has been identified in the thick disc.

One remaining issue that is not simply solved by this scenario is the mass budget problem, as the FG/SG ratio in the stellar populations below $[\mathrm{Fe} / \mathrm{H}] \lesssim-1$ is strongly constrained by the bulge MDF discussed in Section 3.6. This problem could possibly be circumvented if molecular clouds formed stars in a range of $[\mathrm{Fe} / \mathrm{H}]$, which might make possible pollution of the material going into the formation of metal-poor N-rich stars by the ejecta of their more metal-rich counterparts. Admittedly, this seems a bit contrived, but perhaps not entirely outside the realm of possibilities, since evidence for self-enrichment in star-forming regions has been found before (Cunha \& Lambert 1992). Assuming that there is any physical reality to these speculations, one could conceivably devise differences between the abundance patterns of field and GC N-rich stars that would hint at their origin, and possibly help constrain the models for the formation of both stellar populations.

\subsubsection{Oldest stars}

We conclude our exploration of scenarios that may explain the origin of $\mathrm{N}$-rich stars by briefly mentioning another possible interpretation not associated with a GC origin. For a more detailed discussion, we refer the readers to a forthcoming paper (Chiappini et al., in preparation). This scenario advocates that $\mathrm{N}$-rich stars are among the oldest in the Galaxy and their abundances are in fact the imprints of the very early chemical enrichment by the first stellar generations, which polluted the interstellar medium prior to the formation of GCs. Some numerical simulations predict that the oldest stars in the Galaxy are indeed to be found in its central regions (e.g. Brook et al. 2007; Tumlinson 2010). In the Galactic halo, these very early phases of chemical enrichment are traced by halo stars with $[\mathrm{Fe} / \mathrm{H}] \lesssim-2.5$. In the central regions of the Galaxy however, the star formation rate is believed to have been higher, so that the oldest stars in the bulge would have $[\mathrm{Fe} / \mathrm{H}] \sim-1$ (e.g. Barbuy et al. 2009, 2014; Chiappini et al. 2011) which is around the metal-poor tail of the old bulge MDF, and also where the N-rich MDF peaks (Section 3.6). According to this view, N-rich stars may be opening a window into the initial stages of the formation of the Galaxy, which is of course a very exciting prospect.

There are similarities between the abundance patterns of N-rich stars and model predictions for the oldest stars. For instance, models based on enrichment by fast rotating stars (the so-called 'spinstars'; see Chiappini 2013, for a review) predict an enhancement in ${ }^{14} \mathrm{~N}$ and ${ }^{13} \mathrm{C}$, a correlation between $[\mathrm{N} / \mathrm{Fe}]$ and $[\mathrm{Al} / \mathrm{Fe}]$, and a modest enhancement in ${ }^{12} \mathrm{C}$, as well as some contribution to the s-process nucleosynthesis. While the nitrogen enhancement and its correlation with aluminium are corroborated by the data, observations at face value are at odds with predictions for carbon, which is depleted and in fact anticorrelated with nitrogen in the N-rich sample. For the
$\mathrm{N}$-rich stars from our sample, it is hard to assess without detailed modelling of the impact of mixing whether the latter disagreement is real or due to stellar evolution effects. On the other hand, recall that we removed stars with $[\mathrm{C} / \mathrm{Fe}]>+0.15$ from consideration, which obviously biased our sample against stars with strong carbon enhancements. Determination of s-process element abundances for a sample of $\mathrm{N}$-rich stars including some with high $[\mathrm{C} / \mathrm{Fe}]$ would provide a good test of those model predictions.

\subsubsection{Final considerations}

Without further information, it is impossible to decide which of the above scenarios is the most likely to account for the discovery reported in this paper. The one that perhaps is the least favoured by the data is that proposing that N-rich stars are the oldest in the Galaxy, in view of the prediction of enhanced carbon abundances, which is not verified by the data. On the other hand, this scenario may not suffer from the mass budget problem. It is however conceivable that all these channels have contributed to the formation of the N-rich stellar population present in the inner Galaxy.

The in situ and ex situ GC channels described above assume that all N-rich stars were necessarily formed in Galactic GCs and were later lost to the field when the GCs were ultimately disrupted. Each channel likens the newly found stars to existing Galactic GCs associated with the disc/bulge (in situ) and halo (ex situ) components of the Galaxy. In the shared nursery scenario, there should be a similar balance between an in situ and an ex situ origin for the N-rich stars currently found inhabiting the inner Galaxy. That is because in this scenario N-rich stars are proposed to have been gestated in similar molecular clouds as GCs, thus sharing their environmental origin. On the other hand, the 'oldest stars' channel requires these stars to have been formed in situ, as only in the deep gravitational potential of the Galaxy could star formation be intense enough to generate stars with $[\mathrm{Fe} / \mathrm{H}] \sim-1$ in a very short time.

We hypothesize that some yet unknown fraction of the $[\mathrm{Fe} / \mathrm{H}]<-1 \mathrm{~N}$-rich stars found in the inner Galaxy originated ex situ, whereas the metal-rich component and the remainder of the metal-poor stars would have been formed in situ with perhaps some contribution by star formation at very early stages. The term in situ here means different things for these two latter populations, as dissolved GCs are proposed to have been formed in an early turbulent disc and later migrated to the inner Galaxy (Shapiro et al. 2010), whereas the 'oldest stars' are proposed to having been formed in the bulge. A more detailed analysis of an enlarged sample, including further elemental abundances, will surely provide further insights into how the parent systems were formed, potentially destroyed, and eventually left these trace populations as remnants in the inner Galaxy, and how much stellar mass was contributed by the mechanisms put forward here - or by other unforeseen means. By tracing back the steps that brought these populations to their current configuration, we expect to gain a deeper understanding of the processes leading to the formation of GCs, and of the Galactic bulge itself.

\section{SUMMARY}

The main conclusions of this paper are the following.

(i) We have discovered a number of field stars in the inner Galaxy with high [N/Fe], which in addition is anticorrelated with the abundance of carbon and correlated with that of aluminium. This 
abundance pattern is characteristic of so-called ' $\mathrm{SG}$ ' $\mathrm{GC}$ stellar populations.

(ii) The lower limit of the stellar mass ascribed to this new stellar population with a GC-like abundance pattern exceeds that of the existing GCs by a factor of $\sim 6-8$. If these stars are assumed to be the by-products of the destruction of old GCs, our result implies that the Galactic GCs are the remnants of a much larger system that was largely destroyed. If the GC origin of the N-rich stars is confirmed, the location of the Galaxy very near the mean ratio $\eta$ between integrated GC mass and total mass from Hudson et al. (2014) suggests that vigorous GC destruction is a universal process. That notion is further corroborated, although tentatively, by similarities between the mean abundance patterns in the cores of early-type galaxies and those of N-rich stars. It is striking that such a large destruction rate is so finely tuned across a wide range of masses.

(iii) Again assuming that $\mathrm{N}$-rich stars result from GC dissolution, we derive an upper limit of $\sim 93$ per cent for the fraction of GC mass in the form of stars with an FG abundance pattern. This result challenges models of chemical evolution of GCs that postulate larger fractions. It makes it quite difficult, perhaps impossible, to solve the mass budget problem without revising yields from stellar evolution models. Failing that, the whole notion that 'SG' stars are connected to their 'FG' counterparts by chemical evolution through incorporation of by-products of stellar nucleosynthesis may have to be altogether dropped. That is a riveting prospect.

(iv) Under reasonable assumptions for the primordial ratio between FG and SG stars, the contribution of dissolved GCs to the mass of the Galactic bulge is estimated to be small, of the order of a few per cent. Given the spatial overlap of all Galactic components within the central few kpc of the Galaxy, a definition regarding whether N-rich stars belong to the halo, the thick disc, or other components is impossible. The evidence discussed in this paper favours an association with the inner halo of the Galaxy, although this conclusion is by no means definitive. If $\mathrm{N}$-rich stars indeed belong to the halo, they contribute a minimum of 19-25 per cent to the stellar mass in its inner $\sim 2 \mathrm{kpc}$. An accurate estimate is hampered by uncertainties in quantities such as the FG/SG ratio, the contribution of the halo to the stellar mass in the inner Galaxy, and the fraction of N-rich stars that are associated with the inner halo. Nevertheless, this mass fraction exceeds that found in other studies for the outer halo by a factor of several.

(v) The MDF of the newly discovered stellar population does not match that of the bulk of the Galactic bulge. This result suggests that the bulge cannot have been built from a population of dissolved GCs, unless special assumptions are made regarding the incidence of $\mathrm{N}$-rich populations in metal-rich GCs, which are not in agreement with current observational evidence. We conclude that destruction and/or evaporation of GCs accounts for no more than a few per cent of the stellar mass of the Galactic bulge.

(vi) The MDF of the newly discovered stars does not match that of the Galactic GC populations. This result suggests that the $\mathrm{N}$-rich stellar population discovered in the inner Galaxy does not result from simple 'thinning' of the existing Galactic GC population through mass-loss from tidal evaporation. It may also imply that these stars have never been gravitationally bound to any GC.

(vii) We hypothesize that $\mathrm{N}$-rich stars may have resulted from four possible mechanisms. The first two are closely related and involve the destruction of GC populations formed in situ and ex situ. A third mechanism contends that $\mathrm{N}$-rich stars (and presumably most field stars with a so-called SG abundance pattern) were not perforce associated with Galactic GCs, and perhaps most of them may have been formed in similar environments, while never being gravitationally bound to the GCs themselves. A fourth mechanism, namely a very early star formation from a medium polluted by 'spinstars', matches some of the data at least qualitatively, but more work needs to be done to put its predictions to test.

(viii) Regardless of their origin, we find that the vast majority of stars with an SG abundance pattern today live in the field, not in GCs. If indeed these newly discovered stars were never associated with any $\mathrm{GC}$, it is conceivable that the abundance patterns of these "field SG stars' may differ in detail from those of their GC counterparts, in ways that need to be theoretically devised and observationally verified.

(ix) We emphasize that there are a number of key pieces of evidence that are difficult to understand, including the absence, in our sample, of N-rich stars in the Galactic disc, the MDF differences between N-rich stars and the Galactic GC system, and whether the relative numbers of $\mathrm{N}$-rich stars in the inner and outer halo can be reconciled within a single formation scenario. The ultimate association of the N-rich stars with their counterparts currently living in Galactic GCs also requires the determination of other elemental abundances, such as $\mathrm{Na}$ and s-process elements. Detailed modelling and more extended observations will hopefully address these questions.

The results presented herein provide eloquent confirmation of the power of high-resolution spectroscopy applied to large stellar samples to provide key insights into the history of formation of the Galaxy. In the $H$ band, the added benefit of low extinction and relatively easy access to lines of $\mathrm{CN}, \mathrm{CO}$, and $\mathrm{OH}$ make APOGEE a powerful tool to identify remnants of GC disruption at low Galactic latitudes. Exploration of methods to constrain the contribution by various parent systems to the stellar field of the Galaxy is a growing field, which we expect will flourish within the next several years, with the delivery of larger stellar samples, with even better and more detailed phase space and chemical information.

\section{ACKNOWLEDGEMENTS}

RPS thanks Diederik Kruijssen, Alessio Mucciarelli, Carmela Lardo, Maurizio Salaris, Rob Crain, Renyue Cen, Jenny Greene, David Spergel, David Nataf, and Jakob Walcher for enlightening discussions and/or comments on an early version of this manuscript, and Ingrid \& Oliver for everything.

Funding for SDSS-III has been provided by the Alfred P. Sloan Foundation, the Participating Institutions, the National Science Foundation, and the US Department of Energy Office of Science. The SDSS-III website is http://www.sdss3.org/. SDSS-III is managed by the Astrophysical Research Consortium for the Participating Institutions of the SDSS-III Collaboration including the University of Arizona, the Brazilian Participation Group, Brookhaven National Laboratory, University of Cambridge, Carnegie Mellon University, University of Florida, the French Participation Group, the German Participation Group, Harvard University, the Instituto de Astrofísica de Canarias, the Michigan State/Notre Dame/JINA Participation Group, Johns Hopkins University, Lawrence Berkeley National Laboratory, Max Planck Institute for Astrophysics, New Mexico State University, New York University, Ohio State University, Pennsylvania State University, University of Portsmouth, Princeton University, the Spanish Participation Group, University of Tokyo, University of Utah, Vanderbilt University, University of Virginia, University of Washington, and Yale University. TCB 
acknowledges partial support for this work from grants PHY 0822648; Physics Frontier Center/Joint Institute or Nuclear Astrophysics (JINA), and PHY 14-30152; Physics Frontier Center/JINA Center for the Evolution of the Elements (JINA-CEE), awarded by the US National Science Foundation. RC acknowledges support provided by the Spanish Ministry of Economy and Competitiveness under grants AYA2010-16717 and AYA2013-42781P. CAP is thankful for support from the Spanish Ministry of Economy and Competitiveness (MINECO) through grant AYA201456359-P. SLM acknowledges the support of the Australian Research Council through DECRA Fellowship DE140100598. SM has been supported by the János Bolyai Research Scholarship of the Hungarian Academy of Sciences. DAGH was funded by the Ramón y Cajal fellowship number RYC-2013-14182. DAGH and OZ acknowledge support provided by the MINECO under grant AYA-2014-58082-P.

\section{REFERENCES}

Abate C., Pols O. R., Izzard R. G., Mohamed S. S., de Mink S. E., 2013, A\&A, 552, A26

Abate C., Pols O. R., Stancliffe R. J., Izzard R. G., Karakas A. I., Beers T. C., Lee Y. S., 2015, A\&A, 581, 62

Abt H. A., Willmarth D. W., 2004, Rev. Mex. Astron. Astrofis. Ser. Conf., 21,37

Ahn C. P. et al., 2014, ApJS, 211, 17

Alam S. et al., 2015, ApJS, 219, 12

Alvarez R., Plez B., 1998, A\&A, 330, 1109

An D., Beers T. C., Santucci R. M., Carollo D., Placco V. M., Lee Y. S., Rossi S., 2015, ApJ, 813, L28

Angelou G. C., Stancliffe R. J., Church R. P., Lattanzio J. C., Smith G. H., 2012, ApJ, 749, 128

Ashman K. M., Zepf S. E., 1992, ApJ, 384, 50

Babusiaux C. et al., 2010, A\&A, 519, A77

Barber C., Starkenburg E., Navarro J. F., McConnachie A. W., Fattahi A., 2014, MNRAS, 437, 959

Barbuy B., Zoccali M., Ortolani S., Hill V., Minniti D., Bica E., Renzini A., Gómez A., 2009, A\&A, 507, 405

Barbuy B. et al., 2014, A\&A, 570, A76

Bastian N., Lardo C., 2015, MNRAS, 453, 357

Bastian N., Lamers H. J. G. L. M., de Mink S. E., Longmore S. N., Goodwin S. P., Gieles M., 2013, MNRAS, 436, 2398

Bastian N., Cabrera-Ziri I., Salaris M., 2015, MNRAS, 449, 3333

Baumgardt H., Makino J., 2003, MNRAS, 340, 227

Bekki K., 2011, MNRAS, 412, 2241

Bekki K., 2012, ApJ, 748, 78

Bell R. A., Tripicco M. J., 1991, AJ, 102, 777

Belokurov V., Evans N. W., Irwin M. J., Hewett P. C., Wilkinson M. I., 2006a, ApJ, 637, L29

Belokurov V. et al., 2006b, ApJ, 642, L137

Binney J. et al., 2014, MNRAS, 437, 351

Blitz L., Spergel D. N., 1991, ApJ, 379, 631

Blumenthal G. R., Faber S. M., Primack J. R., Rees M. J., 1984, Nature, 311,517

Bournaud F., 2016, Astrophysics and Space Science Library, Vol. 418, Galactic Bulges. Springer, Berlin, p. 355

Bournaud F., Elmegreen B. G., Elmegreen D. M., 2007, ApJ, 670, 237

Brandt T. D., Kocsis B., 2015, ApJ, 812, 15

Bressan A., Marigo P., Girardi L., Salasnich B., Dal Cero C., Rubele S., Nanni A., 2012, MNRAS, 427, 127

Brook C. B., Kawata D., Scannapieco E., Martel H., Gibson B. K., 2007, ApJ, 661, 10

Brown T. M., Ferguson H. C., Davidsen A. F., Dorman B., 1997, ApJ, 482, 685

Brown T. M., Ferguson H. C., Stanford S. A., Deharveng J.-M., 1998, ApJ, 504,113
Brown T. M., Bowers C. W., Kimble R. A., Sweigart A. V., Ferguson H. C., 2000, ApJ, 532, 308

Burstein D., Faber S. M., Gaskell C. M., Krumm N., 1984, ApJ, 287, 586

Cabrera-Lavers A., Garzón F., 2003, A\&A, 403, 383

Cabrera-Ziri I. et al., 2015, MNRAS, 448, 2224

Carollo D. et al., 2007, Nature, 450, 1020

Carollo D. et al., 2010, ApJ, 712, 692

Carollo D., Martell S. L., Beers T. C., Freeman K. C., 2013, ApJ, 769, 87

Carretta E., Bragaglia A., Gratton R., Lucatello S., 2009, A\&A, 505, 139

Carretta E., Bragaglia A., Gratton R. G., Recio-Blanco A., Lucatello S., D’Orazi V., Cassisi S., 2010, A\&A, 516, A55

Catelan M. et al., 2014, in Guzik J. A., Chaplin W. J., Handler G., Pigulski A., eds, Proc. IAU Symp. 301, Precision Asteroseismology. Cambridge Univ. Press, Cambridge, p. 395

Chabrier G., 2003, PASP, 115, 763

Charbonnel C., Lagarde N., 2010, A\&A, 522, A10

Chiappini C., 2013, Astron. Nachr., 334, 595

Chiappini C., Frischknecht U., Meynet G., Hirschi R., Barbuy B., Pignatari M., Decressin T., Maeder A., 2011, Nature, 472, 454

Cioni M.-R. et al., 2000, A\&AS, 144, 235

Code A. D., Welch G. A., 1979, ApJ, 228, 95

Conroy C., 2012, ApJ, 758, 21

Conroy C., Spergel D. N., 2011, ApJ, 726, 36

Conroy C., Graves G. J., van Dokkum P. G., 2014, ApJ, 780, 33

Cunha K., Lambert D. L., 1992, ApJ, 399, 586

D’Ercole A., Vesperini E., D’Antona F., McMillan S. L. W., Recchi S., 2008, MNRAS, 391, 825

Da Costa G. S., Cottrell P. L., 1980, ApJ, 236, L83

Deason A. J., Belokurov V., Weisz D. R., 2015, MNRAS, 448, L77

Decressin T., Meynet G., Charbonnel C., Prantzos N., Ekström S., 2007, A\&A, 464, 1029

Dékány I., Minniti D., Catelan M., Zoccali M., Saito R. K., Hempel M., Gonzalez O. A., 2013, ApJ, 776, L19

Dekel A. et al., 2009, Nature, 457, 451

Dorman B., O'Connell R. W., Rood R. T., 1995, ApJ, 442, 105

Duquennoy A., Mayor M., 1991, A\&A, 248, 485

Eggen O., Lynden-Bell D., Sandage A. R., 1962, ApJ, 136, 748

Eisenstein D. J. et al., 2011, AJ, 142, 72

Elmegreen B. G., Hunter D. A., 2010, ApJ, 712, 604

Elmegreen B. G., Bournaud F., Elmegreen D. M., 2008, ApJ, 688, 67

Epchtein N. et al., 1997, The Messenger, 87, 27

Fattahi A. et al., 2016, MNRAS, 457, 844

Fernández-Alvar E. et al., 2015, A\&A, 577, A81

Fernández-Trincado J. G. et al., 2016, ApJL, preprint (arXiv:1604.01279)

Fiorentino G. et al., 2015, ApJ, 798, L12

Fischer D. A., Marcy G. W., 1992, ApJ, 396, 178

Freeman K. H., Bland-Hawthorn J., 2002, ARA\&A, 40, 487

Freeman K. E. et al., 2013, MNRAS, 428, 3660

Fulbright J. P., McWilliam A., Rich R. M., 2007, ApJ, 661, 1152

García Pérez A. E. et al., 2016, AJ, 151, 144

García-Lario P., Manchado A., Pych W., Pottasch S. R., 1997, A\&AS, 126, 479

Gilbert K. M. et al., 2014, ApJ, 796, 76

Gilmore G. et al., 2012, The Messenger, 147, 25

Girardi L., Bressan A., Bertelli G., Chiosi C., 2000, A\&AS, 141, 371

Girardi L. et al., 2010, ApJ, 724, 1030

Gnedin O. Y., Ostriker J. P., Tremaine S., 2014, ApJ, 785, 71

Gratton R. G., Sneden C., Carretta E., Bragaglia A., 2000, A\&A, 354, 169

Gratton R., Sneden C., Carretta E., 2004, ARA\&A, 42, 385

Gratton R. G., Carretta E., Bragaglia A., 2012, A\&AR, 20, 50

Graves G. J., Faber S. M., Schiavon R. P., Yan R., 2007, ApJ, 671, 243

Gray R. O., Corbally C. J., 2009, Stellar Spectral Classification. Princeton Univ. Press, Princeton, NJ

Greggio L., Renzini A., 1999, Mem. Soc. Astron. Ital., 70, 691

Gunn J. E. et al., 2006, AJ, 131, 2332

Gustafsson B., Edvardsson B., Eriksson K., Jørgensen U. G., Nordlund Å., Plez B., 2008, A\&A, 486, 951

Habing H. J., 1996, A\&AR, 7, 97 
Harris W. E., 1996, AJ, 112, 1487

Hesser J. E., Bell R. A., Harris G. L. H., Cannon R. D., 1982, AJ, 87, 1470

Hill V. et al., 2011, A\&A, 534, A80

Holtzman J. A. et al., 2015, AJ, 150, 148

Hooper D., Goodenough L., 2011, Phys. Lett. B, 697, 412

Hopkins P. F., 2014, ApJ, 797, 59

Hudson M. J., Harris G. L., Harris W. E., 2014, ApJ, 787, L5

Ibata R. A., Gilmore G., Irwin M. J., 1994, Nature, 370, 194

Ibata R. A. et al., 2014, ApJ, 780, 128

Iben I., Jr, Renzini A., 1983, ARA\&A, 21, 271

Indebetouw R. et al., 2005, ApJ, 619, 931

Jiménez-Esteban F. M., García-Lario P., Engels D., Manchado A., 2006, A\&A, 458, 533

Johnson C. I., Rich R. M., Fulbright J. P., Valenti E., McWilliam A., 2011, ApJ, 732, 108

Johnson C. I., Rich R. M., Kobayashi C., Kunder A., Koch A., 2014, AJ, 148,67

Karakas A. I., Lattanzio J. C., 2014, PASA, 31, e030

Karinkuzhi D., Goswami A., 2015, MNRAS, 446, 2348

Koesterke L., 2009, in Hubeny I., Stone J. M., MacGregor K., Werner K., eds, AIP Conf. Ser. Vol. 1171, Recent Directions in Astrophysical Quantitative Spectroscopy and Radiation Hydrodynamics. Am. Inst. Phys., New York, p. 73

Koornneef J., 1983, A\&A, 128, 84

Kraft R. P., Sneden C., Smith G. H., Shetrone M. D., Langer G. E., Pilachowski C. A., 1997, AJ, 113, 279

Kravtsov A. V., Gnedin O. Y., 2005, ApJ, 623, 650

Kroupa P., 2001, MNRAS, 322, 231

Kruijssen J. M. D., 2014, Class. Quantum Grav., 31, 244006

Kruijssen J. M. D., 2015, MNRAS, 454, 1658

Kruijssen J. M. D., Portegies Zwart S. F., 2009, ApJ, 698, L158

Kruijssen J. M. D., Pelupessy F. I., Lamers H. J. G. L. M., Portegies Zwart S. F., Icke V., 2011, MNRAS, 414, 1339

Kruijssen J. M. D., Pelupessy F. I., Lamers H. J. G. L. M., Portegies Zwart S. F., Bastian N., Icke V., 2012, MNRAS, 421, 1927

Kurucz R. L., 1993, Kurucz CD-ROM No. 13, Smithsonian Astrophysical Observatory, Cambridge, MA

Lardo C., Pancino E., Mucciarelli A., Milone A. P., 2012, A\&A, 548, A107

Larsen S. S., Strader J., Brodie J. P., 2012, A\&A, 544, L14

Larsen S. S., Brodie J. P., Forbes D. A., Strader J., 2014, A\&A, 565, A98

Law D. R., Majewski S. R., 2010, ApJ, 718, 1128

Lawrence A. et al., 2007, MNRAS, 379, 1599

Lind K. et al., 2015, A\&A, 575, L12

Longmore S. N. et al., 2014, Protostars and Planets VI. Univ. Arizona Press, Tucson, p. 291

Lucatello S., Tsangarides S., Beers T. C., Carretta E., Gratton R. G., Ryan S. G., 2005a, ApJ, 625, 825

Lucatello S., Gratton R. G., Beers T. C., Carretta E., 2005b, ApJ, 625, 833

McCarthy I. G., Font A. S., Crain R. A., Deason A. J., Schaye J., Theuns T., 2012, MNRAS, 420, 2245

McClure R. D., Woodsworth A. W., 1990, ApJ, 352, 709

McWilliam A., Rich R. M., 1994, ApJS, 91, 749

McWilliam A., Zoccali M., 2010, ApJ, 724, 1491

Majewski S. R., Zasowski G., Nidever D. L., 2011, ApJ, 739, 25

Majewski S. M. et al., 2016, preprint (arXiv:1509.05420)

Marengo M., Hora J. L., Barmby P., Willner S. P., Allen L. E., Schuster M. T., Fazio G. G., 2007, in Kerschbaum F., Charbonnel C., Wing R. F., eds, ASP Conf. Ser. Vol. 378, Why Galaxies Care About AGB Stars: Their Importance as Actors and Probes. Astron. Soc. Pac., San Francisco, p. 80

Martell S. L., Grebel E. K., 2010, A\&A, 518, A14

Martell S. L., Smith G. H., Briley M. M., 2008, AJ, 136, 2522

Martell S. L., Smolinski J. P., Beers T. C., Grebel E. K., 2011, A\&A, 534, A136

Martell S. L. et al., 2016, ApJ, 825, 146

Mészáros Sz. et al., 2012, AJ, 144, 120

Mészáros Sz. et al., 2013, AJ, 146, 133

Mészáros Sz. et al., 2015, AJ, 149, 153

Monet D. G. et al., 2003, AJ, 125, 984
Muratov A. L., Gnedin O. Y., 2010, ApJ, 718, 1266

Nataf D. M., Udalski A., Gould A., Fouqué P., Stanek K. Z., 2010, ApJ, 721, L28

Ness M., Freeman K., Athanassoula E., 2012, ApJ, 756, 22

Ness M., Freeman K., Athanassoula E., 2013a, MNRAS, 430, 836

Ness M., Freeman K., Athanassoula E., 2013b, MNRAS, 432, 2092

Ness M. et al., 2016, ApJ, 819, 2

Nidever D. L. et al., 2015, AJ, 150, 173

Norris J., Cottrell P. L., 1979, ApJ, 229, L69

Norris J., Zinn R., 1977, ApJ, 215, 74

Norris J., Cottrell P. L., Freeman K. C., Da Costa G. S., 1981, ApJ, 244, 205

O'Connell R. W., 1999, ARA\&A, 37, 603

Odenkirchen M. et al., 2003, AJ, 126, 2385

Plez B., 2012, Astrophysics Source Code Library, record ascl:1205.004

Plez B., Brett J. M., Nordlund A., 1992, A\&A, 256, 551

Portail M., Wegg C., Gerhard O., Martinez-Valpuesta I., 2015, MNRAS, 448,713

Ramírez S. V., Arendt R. G., Sellgren K., Stolovy S. R., Cotera A., Smith H. A., Yusef-Zadeh F., 2008, ApJS, 175, 147

Renzini A., 2008, MNRAS, 391, 354

Renzini A., Voli M., 1981, A\&A, 94, 175

Robin A. C., Marshall D. J., Schultheis M., Reylé C., 2012, A\&A, 538, A106

Robin A. C., Reylé C., Fliri J., Czekaj M., Robert C. P., Martins A. M. M., 2014, A\&A, 569, A13

Rockosi C. M. et al., 2002, AJ, 124, 349

Rojas-Arriagada A. et al., 2014, A\&A, 569, A103

Rossi L. J., Hurley J. R., 2015, MNRAS, 446, 3389

Russell H. N., 1934, ApJ, 79, 317

Ryde N., Schultheis M., Grieco V., Matteucci F., Rich R. M., Uttenthaler S., 2016, AJ, 151, 1

Saito R. K. et al., 2012, A\&A, 537, A107

Salpeter E. E., 1955, ApJ, 121, 161

Schaerer D., Charbonnel C., 2011, MNRAS, 413, 2297

Schiavon R. P., 2007, ApJS, 171, 146

Schiavon R. P., Barbuy B., Bruzual A. G., 2000, ApJ, 532, 453

Schiavon R. P., Caldwell N., Morrison H., Harding P., Courteau S., MacArthur L. A., Graves G. J., 2012a, AJ, 143, 14

Schiavon R. P. et al., 2012b, AJ, 143, 121

Schiavon R. P., Caldwell N., Conroy C., Graves G. J., Strader J., MacArthur L. A., Courteau S., Harding P., 2013, ApJ, 776, L7

Schönrich R., Binney J., Asplund M., 2012, MNRAS, 420, 1281

Schönrich R., Asplund M., Casagrande L., 2014, ApJ, 786, 7

Schultheis M., Lançon A., Omont A., Schuller F., Ojha D. K., 2003, A\&A, 405,531

Searle L., Zinn R., 1978, ApJ, 225, 357

Shapiro K. L., Genzel R., Förster Schreiber N. M., 2010, MNRAS, 403, L36

Shetrone M. et al., 2015, ApJS, 221, 24

Skrutskie M. F. et al., 2006, AJ, 131, 1163

Smith G. H., Martell S. L., 2003, PASP, 115, 1211

Smith R. J., Alton P., Lucey J. R., Conroy C., Carter D., 2015, MNRAS, 454, L71

Sofue Y., Honma M., Omodaka T., 2009, PASJ, 61, 227

Soszyński I. et al., 2013, Acta Astron., 63, 21

Spergel D. N. et al., 2003, ApJS, 148, 175

Spinrad H., Taylor B. J., 1971, ApJS, 22, 445

Starkenburg E., Shetrone M. D., McConnachie A. W., Venn K. A., 2014 , MNRAS, 441, 1217

Strader J. et al., 2013, ApJ, 775, L6

Ting Y.-S., Conroy C., Goodman A., 2015, ApJ, 807, 104

Ting Y.-S., Conroy C., Rix H.-W., 2016, ApJ, 816, 10

Tissera P., Beers T. C., Carollo D., Scannapieco C., 2014, MNRAS, 439, 2128

Tonini C., 2013, ApJ, 762, 39

Tremaine S. D., Ostriker J. P., Spitzer L., Jr1975, ApJ, 196, 407

Tsuji T., 1973, A\&A, 23, 411

Tumlinson J., 2010, ApJ, 708, 1398

Udalski A., Kubiak M., Szymanski M., 1997, Acta Astron., 47, 319

van Dokkum P. G., Conroy C., 2010, Nature, 468, 940 
Ventura P., Di Criscienzo M., Carini R., D’Antona F., 2013, MNRAS, 431, 3642

White S. D. M., Rees M. J., 1978, MNRAS, 183, 341

Worthey G., Tang B., Serven J., 2014, ApJ, 783, 20

Wray J. J., Eyer L., Paczyński B., 2004, MNRAS, 349, 1059

Yi S. K. et al., 2005, ApJ, 619, L111

Yoon S.-J., Yi S. K., Lee Y.-W., 2006, Science, 311, 1129

Zamora O. et al., 2015, AJ, 149, 181

Zasowski G. et al., 2013, AJ, 146, 81

Zieleniewski S., Houghton R. C. W., Thatte N., Davies R. L.2015, MNRAS, 452,597

Zoccali M., Hill V., Lecureur A., Barbuy B., Renzini A., Minniti D., Gómez A., Ortolani S., 2008, A\&A, 486, 177

\section{SUPPORTING INFORMATION}

Additional Supporting Information may be found in the online version of this article:

bulge_sample_paper.fits

M3_sample_paper.fits

\section{M5_sample_paper.fits \\ M71_sample_paper.fits \\ M107_sample_paper.fits \\ N6760_sample_paper.fits}

(http://www.mnras.oxfordjournals.org/lookup/suppl/doi:10.1093/ mnras/stw2162/-/DC1).

Please note: Oxford University Press is not responsible for the content or functionality of any supporting materials supplied by the authors. Any queries (other than missing material) should be directed to the corresponding author for the article.

\section{APPENDIX A: N-RICH STARS}

In Table A1, we provide the identities of our sample of 58 stars identified as N-rich in the inner Galaxy by APOGEE, along with parameters employed in the analysis.

Table A1. N-rich stars identified in the inner Galaxy.

\begin{tabular}{|c|c|c|c|c|c|c|c|c|c|c|}
\hline APOGEE_ID & $\alpha_{2000}$ & $\delta_{2000}$ & $\mathrm{~S} / \mathrm{N}$ & $T_{\text {eff }}$ & $\log g$ & {$[\mathrm{Fe} / \mathrm{H}]$} & {$[\mathrm{Al} / \mathrm{Fe}]$} & {$[\mathrm{C} / \mathrm{Fe}]$} & {$[\mathrm{N} / \mathrm{Fe}]$} & $d(\mathrm{kpc})$ \\
\hline 2M16514646-2127071 & 52.943621 & -21.451977 & 121 & & 1.7 & $-0.86 \pm 0.04$ & $0.36 \pm 0.13$ & $-0.21 \pm 0.08$ & $0.10 \pm 0.11$ & $5.3 \pm 1.3$ \\
\hline 2M17134700-2441353 & 258.445841 & -24.693153 & 133 & 4298 & 0.9 & $-1.55 \pm 0.05$ & $0.35 \pm 0.20$ & $-0.42 \pm 0.12$ & $0.82 \pm 0.15$ & $8.0 \pm 1.3$ \\
\hline 2M17161691-2458586 & 59.070484 & -24.982969 & 158 & 4133 & 1.3 & $-0.10 \pm 0.03$ & $0.34 \pm 0.06$ & $-0.30 \pm 0.04$ & $0.59 \pm 0.07$ & \\
\hline 2M17173203-2439094 & 259.383468 & -24.65262 & 223 & & -0.2 & $-0.78 \pm 0.04$ & $0.28 \pm 0.08$ & $0.34 \pm 0.05$ & $0.58 \pm 0.08$ & \\
\hline 2M17211817-2735530 & 260.325717 & -27.598082 & 228 & 3736 & 0.4 & $-0.41 \pm 0.03$ & $0.27 \pm 0.06$ & $-0.24 \pm 0.04$ & $0.60 \pm 0.07$ & $1 \pm 1.1$ \\
\hline 2M17263951-2406247 & 261.664646 & -24.106882 & 126 & 40 & 0.9 & $-0.50 \pm 0.04$ & $-0.00 \pm 0.08$ & $-0.13 \pm 0.05$ & $0.52 \pm 0.08$ & $9.0 \pm 2.0$ \\
\hline 2M17271907-2718040 & 61.829481 & -27.301126 & 148 & 41 & 1.5 & $-0.54 \pm 0.04$ & $0.25 \pm 0.08$ & $-0.20 \pm 0.06$ & $0.55 \pm$ & \\
\hline 7303980-2330234 & 262.66 & -23.506 & 240 & & 0. & $-0.95 \pm 0.04$ & 09 & $-0.22 \pm 0.06$ & 09 & \\
\hline & 262.71 & -26.864 & 157 & & & & & & & \\
\hline 2M17333623-2548156 & 263.400967 & -25.80436 & 147 & & 1.0 & $-1.05 \pm 0$ & & $-0.04 \pm 0$ & & $8.2 \pm 1.6$ \\
\hline 289 & 263.7 & -29.541 & 119 & 4247 & 1. & -0.7 & & $-0.23 \pm$ & $0.87 \pm$ & $8.0 \pm 1.9$ \\
\hline 2M17352288-2913255 & 263.845356 & -29.22377 & 194 & & 1.0 & & & $-0.37 \pm$ & & $6.2 \pm 1.2$ \\
\hline 2M17353215-2759106 & 263.88397 & -27.986303 & 116 & & 0.9 & $-0.56 \pm 0$ & & & & $9.2 \pm 1.8$ \\
\hline 2M17354267-2406233 & 263.927815 & -24.106478 & 234 & & 0. & $-0.71 \pm 0.04$ & $0.21 \pm$ & $0.14 \pm 0.05$ & $0.55 \pm 0$. & \pm 1.0 \\
\hline 2M17382269-2748001 & 264.594549 & -27.800049 & 211 & 38 & 0 . & $-1.13 \pm 0.04$ & $0.00 \pm 0.11$ & $-0.36 \pm 0.07$ & $0.81 \pm 0.11$ & $9.0 \pm 0.7$ \\
\hline $2497-3006527$ & 4065 & -30.114656 & 109 & 41 & & -0.8 & 0.32 & -0.10 & 0.86 & 1.3 \\
\hline 2M17390422-2943520 & 264.76 & -29.731115 & 178 & 4047 & 0 . & $-1.20 \pm 0.04$ & $0.44 \pm 0.12$ & $-0.35 \pm 0$ & $0.64 \pm 0.11$ & $7.7 \pm 1.2$ \\
\hline & 265.172631 & -27.249172 & 105 & & 1.3 & & & & & $7.9 \pm 1.9$ \\
\hline 2M17415271-2715374 & 265.469643 & -27.260414 & 151 & 418 & 1.0 & $-1.15 \pm 0.05$ & $0.20 \pm 0.13$ & $-0.35 \pm 0.08$ & $0.90 \pm 0.12$ & $7.3 \pm 1.4$ \\
\hline 2M17431507-2815570 & 265.812795 & -28.26586 & 128 & 417 & 1. & $-0.86 \pm 0.04$ & $-0.67 \pm 0.11$ & $0.01 \pm 0.07$ & $0.50 \pm 0.10$ & $7.5 \pm 1.8$ \\
\hline $2 \mathrm{M} 17442343-2627304$ & 266.097638 & -26.458456 & 184 & & 0 . & $-0.91 \pm 0.04$ & $0.35 \pm 0.10$ & $-0.22 \pm 0.06$ & $0.93 \pm 0.10$ & \\
\hline 2M17453131-2342147 & 266.38 & -23.704111 & 127 & & 1. & $-0.50 \pm 0$ & 0.41 & $0.00 \pm 0$ & 0.81 & \\
\hline 2M17534394-2826411 & 268.433095 & -28.444759 & 182 & 3811 & -0.2 & $-1.01 \pm 0.04$ & $0.21 \pm 0.10$ & $-0.22 \pm 0.06$ & $0.87 \pm 0.10$ & $10.3 \pm 0.7$ \\
\hline 2M17554454-2123058 & 268.93562 & -21.384953 & 159 & 4271 & 1.5 & $-0.62 \pm 0.04$ & $0.24 \pm 0.09$ & $-0.27 \pm 0.06$ & $0.57 \pm 0.09$ & $5.4 \pm 1.5$ \\
\hline
\end{tabular}


Table A1 - continued

\begin{tabular}{lcccccccccc}
\hline APOGEE_ID & \multicolumn{1}{c}{$\alpha_{2000}$} & $\delta_{2000}$ & $\mathrm{~S} / \mathrm{N}$ & $T_{\text {eff }}$ & $\log g$ & {$[\mathrm{Fe} / \mathrm{H}]$} & {$[\mathrm{Al} / \mathrm{Fe}]$} & {$[\mathrm{C} / \mathrm{Fe}]$} & {$[\mathrm{N} / \mathrm{Fe}]$} & $d(\mathrm{kpc})$ \\
\hline 2M18014817-3026237 & 270.450716 & -30.439939 & 117 & 4311 & 1.3 & $-0.93 \pm 0.04$ & $0.43 \pm 0.13$ & $-0.08 \pm 0.08$ & $0.88 \pm 0.11$ & $9.6 \pm 2.1$ \\
2M18020427-1810191 & 270.517792 & -18.171999 & 241 & 3732 & 0.0 & $-0.56 \pm 0.03$ & $0.11 \pm 0.06$ & $-0.65 \pm 0.04$ & $0.71 \pm 0.07$ & $7.0 \pm 0.7$ \\
2M18022530-2928338 & 270.605421 & -29.476059 & 191 & 3844 & 0.6 & $-0.35 \pm 0.03$ & $0.10 \pm 0.06$ & $-0.55 \pm 0.04$ & $1.08 \pm 0.07$ & $6.8 \pm 1.3$ \\
2M18033335-2929122 & 270.888992 & -29.48674 & 95 & 4482 & 1.8 & $-0.92 \pm 0.05$ & $0.82 \pm 0.14$ & $-0.09 \pm 0.09$ & $0.73 \pm 0.12$ & $8.4 \pm 2.0$ \\
2M18035944-2908195 & 270.997669 & -29.138758 & 162 & 3820 & 0.6 & $-0.47 \pm 0.03$ & $0.18 \pm 0.07$ & $-0.09 \pm 0.04$ & $0.57 \pm 0.07$ & $6.6 \pm 1.1$ \\
2M18054875-3122407 & 271.453164 & -31.377975 & 389 & 3829 & -0.2 & $-1.16 \pm 0.04$ & $0.09 \pm 0.11$ & $-0.32 \pm 0.07$ & $0.69 \pm 0.11$ & $9.8 \pm 0.6$ \\
2M18061336-3147053 & 271.555701 & -31.784821 & 182 & 4427 & 1.6 & $-0.57 \pm 0.04$ & $0.05 \pm 0.09$ & $-0.04 \pm 0.06$ & $0.51 \pm 0.09$ & $6.7 \pm 2.1$ \\
2M18090957-1559276 & 272.289877 & -15.991026 & 135 & 3882 & 0.4 & $-0.28 \pm 0.03$ & $0.04 \pm 0.06$ & $-0.26 \pm 0.04$ & $0.52 \pm 0.07$ & $8.7 \pm 1.7$ \\
2M18102953-2707208 & 272.62305 & -27.122459 & 155 & 4115 & 1.2 & $-0.36 \pm 0.04$ & $0.52 \pm 0.07$ & $0.02 \pm 0.05$ & $0.58 \pm 0.08$ & $6.0 \pm 1.4$ \\
2M18120031-1350169 & 273.001326 & -13.838031 & 131 & 4230 & 1.3 & $-0.97 \pm 0.04$ & $1.16 \pm 0.12$ & $0.08 \pm 0.08$ & $0.64 \pm 0.11$ & $6.0 \pm 1.3$ \\
2M18121957-2926310 & 273.081553 & -29.441954 & 193 & 4031 & 0.8 & $-0.91 \pm 0.04$ & $0.36 \pm 0.10$ & $-0.04 \pm 0.06$ & $0.62 \pm 0.10$ & $9.4 \pm 1.7$ \\
2M18124455-2719146 & 273.185633 & -27.32074 & 211 & 3992 & 0.6 & $-1.02 \pm 0.04$ & $0.09 \pm 0.11$ & $-0.22 \pm 0.07$ & $0.55 \pm 0.10$ & $8.0 \pm 1.2$ \\
2M18165340-2017051 & 274.222524 & -20.284777 & 107 & 4029 & 1.1 & $-0.03 \pm 0.03$ & $0.17 \pm 0.06$ & $-0.39 \pm 0.04$ & $0.66 \pm 0.06$ & $5.8 \pm 1.6$ \\
2M18334592-2903253 & 278.441366 & -29.057034 & 154 & 4264 & 1.6 & $-0.78 \pm 0.04$ & $0.65 \pm 0.11$ & $-0.19 \pm 0.07$ & $0.92 \pm 0.10$ & $6.0 \pm 1.8$ \\
2M18372953-2911046 & 279.373046 & -29.18462 & 188 & 4475 & 2.7 & $-1.06 \pm 0.04$ & $0.39 \pm 0.14$ & $-0.35 \pm 0.09$ & $1.00 \pm 0.12$ & $6.1 \pm 1.3$ \\
2M18442352-3029411 & 281.098036 & -30.494764 & 287 & 4073 & 1.0 & $-0.77 \pm 0.04$ & $0.73 \pm 0.09$ & $-0.15 \pm 0.06$ & $0.92 \pm 0.09$ & $5.4 \pm 1.1$ \\
2M18550318-3043368 & 283.763269 & -30.726915 & 71 & 4444 & 2.1 & $-0.93 \pm 0.04$ & $1.06 \pm 0.15$ & $-0.13 \pm 0.09$ & $0.97 \pm 0.13$ & $10.1 \pm 2.3$ \\
\hline
\end{tabular}

${ }^{1}$ Astrophysics Research Institute, Liverpool John Moores University, 146 Brownlow Hill, Liverpool L3 5RF, UK

${ }^{2}$ Instituto de Astrofísica de Canarias, E-38205 La Laguna, Tenerife, Spain ${ }^{3}$ Departamento de Astrofísica, Universidad de La Laguna (ULL), E-38206 La Laguna, Tenerife, Spain

${ }^{4}$ INAF-Osservatorio Astronomico di Padova, Vicolo dell'Osservatorio 5, I-35122 Padova, Italy

${ }^{5}$ Institut Utinam, CNRS UMR6213, Université de Franche-Comté, OSU THETA Franche-Comté-Bourgogne, Observatoire de Besançon, BP 1615, F-25010 Besançon Cedex, France

${ }^{6}$ Max-Planck-Institut fur Astronomie, Königstuhl 17, D-69117 Heidelberg, Germany

${ }^{7}$ School of Physics, University of New South Wales, Sydney, NSW 2052, Australia

${ }^{8}$ National Optical Astronomy Observatories, Tucson, AZ 85719, USA

${ }^{9}$ Steward Observatory, University of Arizona, Tucson, AZ 85721, USA

${ }^{10}$ Consejo Superior de Investigaciones Cientificas (CSIC)

${ }^{11}$ Rudolf Peierls Centre for Theoretical Physics, 1 Keble Road, Oxford OXI $3 N P, U K$

${ }^{12}$ Leibniz-Institut für Astrophysik Potsdam (AIP), An der Sternwarte 16, D-14482 Potsdam, Germany

${ }^{13}$ University of Texas at Austin, McDonald Observatory, Fort Davis, TX 79734, USA

${ }^{14}$ ELTE Gothard Astrophysical Observatory, Szent Imre Herceg st. 112, H-9704 Szombathely, Hungary

${ }^{15}$ Apache Point Observatory, PO Box 59, Sunspot, NM 88349-0059, USA

${ }^{16}$ Sternberg Astronomical Institute, Moscow State University, 119992 Moscow, Russia
${ }^{17}$ Department of Physics and JINA Center for the Origin of the Elements, University of Notre Dame, Notre Dame, IN 46556, USA

${ }^{18}$ Department of Astronomy, University of Virginia, Charlottesville, VA 22904-4325, USA

${ }^{19}$ Observatório Nacional, São Cristóvão, Rio de Janeiro RJ, 20921-400, Brazil

${ }^{20}$ Department of Astronomy, The Ohio State University, Columbus, $\mathrm{OH}$ 43210, USA

${ }^{21}$ Texas Christian University, Fort Worth, TX 76129, USA

${ }^{22}$ Department of Astronomy and Astrophysics, Institute for Gravitation and the Cosmos, The Pennsylvania State University, University Park, PA 16802, USA

${ }^{23}$ New Mexico State University, Las Cruces, NM 88003, USA

${ }^{24}$ Department of Astronomy, University of Michigan, Ann Arbor, MI 48104, USA

${ }^{25}$ Large Synoptic Survey Telescope, 950 North Cherry Ave, Tucson, AZ 85719, USA

${ }^{26}$ Steward Observatory, 933 North Cherry Ave, Tucson, AZ 85719, USA

${ }^{27}$ Dunlap Institute for Astronomy and Astrophysics, University of Toronto, Toronto, Ontario M5S 3H4, Canada

${ }^{28}$ Laboratoire Lagrange (UMR7293), Universite de Nice Sophia Antipolis, CNRS, Observatoire de la Côte d'Azur, BP 4229, F-06304 Nice Cedex 4, France

${ }^{29}$ Department of Physics and Astronomy, Johns Hopkins University, Baltimore, MD 21218, USA

This paper has been typeset from a $\mathrm{T}_{\mathrm{E}} \mathrm{X} / \mathrm{L} \mathrm{T}_{\mathrm{E}} \mathrm{X}$ file prepared by the author. 